\title{
Initial traces and solvability of Cauchy problem to a semilinear parabolic system
}

\author{
Yohei Fujishima and Kazuhiro Ishige
}

\begin{abstract}
Let $(u, v)$ be a solution to a semilinear parabolic system

$$
(\mathrm{P}) \quad\left\{\begin{array}{lll}
\partial_{t} u=D_{1} \Delta u+v^{p} & \text { in } \quad \mathbf{R}^{N} \times(0, T), \\
\partial_{t} v=D_{2} \Delta v+u^{q} & \text { in } \mathbf{R}^{N} \times(0, T), \\
u, v \geq 0 & \text { in } \mathbf{R}^{N} \times(0, T), \\
(u(\cdot, 0), v(\cdot, 0))=(\mu, \nu) & \text { in } \quad \mathbf{R}^{N},
\end{array}\right.
$$
\end{abstract}

where $N \geq 1, T>0, D_{1}>0, D_{2}>0,0<p \leq q$ with $p q>1$ and $(\mu, \nu)$ is a pair of Radon measures or nonnegative measurable functions in $\mathbf{R}^{N}$. In this paper we study qualitative properties of the initial trace of the solution $(u, v)$ and obtain necessary conditions on the initial data $(\mu, \nu)$ for the existence of solutions to problem $(\mathrm{P})$.

\section{Addresses:}

Y. F.: Department of Mathematical and Systems Engineering, Faculty of Engineering, Shizuoka University, 3-5-1, Johoku, Hamamatsu, 432-8561, Japan.

E-mail: fujishima@shizuoka.ac.jp

K. I.: Graduate School of Mathematical Sciences, The University of Tokyo, 3-8-1 Komaba, Meguro-ku, Tokyo 153-8914, Japan.

E-mail: ishige@ms.u-tokyo.ac.jp 


\section{Introduction}

Consider a semilinear parabolic system

$$
\begin{cases}\partial_{t} u=D_{1} \Delta u+v^{p} & \text { in } \quad \mathbf{R}^{N} \times(0, T), \\ \partial_{t} v=D_{2} \Delta v+u^{q} & \text { in } \quad \mathbf{R}^{N} \times(0, T), \\ u, v \geq 0 & \text { in } \quad \mathbf{R}^{N} \times(0, T),\end{cases}
$$

where $N \geq 1, T>0, D_{1}>0, D_{2}>0$ and $0<p \leq q$ with $p q>1$. Parabolic system (1.1) is one of the simplest parabolic systems and it is an example of reaction-diffusion systems describing heat propagation in a two component combustible mixture. It has been studied extensively in many papers from various points of view, see e.g. [3, 12, 13, 15, 16, 22, 25, 27, 29, 31]. See also [28, Chapter 32] and references therein. In this paper we prove the existence and the uniqueness of the initial trace of a solution $(u, v)$ to parabolic system (1.1) and study qualitative properties of the initial traces. Furthermore, we obtain necessary conditions on the existence of the solutions to problem (1.1) with

$$
(u(\cdot, 0), v(\cdot, 0))=(\mu, \nu) \text { in } \mathbf{R}^{N},
$$

where $(\mu, \nu)$ is a pair of (nonnegative) Radon measures or nonnegative measurable functions in $\mathbf{R}^{N}$.

The study of the initial traces of the solutions to parabolic equations is a classical subject and it has been investigated for various parabolic equations, for example, the heat equation (see [4, 30]), the porous medium equation (see [5, 7, 18]), the parabolic $p$-Laplace equation (see [10, 11]), the doubly nonlinear parabolic equation (see [21, 23, 33]), the fractional diffusion equation (see [9]), the Finsler heat equation (see [1]) and parabolic equations with nonlinear terms (see e.g. [2, 6, 8, 19, 20, 26, 32]). For the semilinear heat equation

$$
\left\{\begin{array}{lll}
\partial_{t} u=\Delta u+u^{p} & \text { in } & \mathbf{R}^{N} \times(0, T), \\
u \geq 0 & \text { in } & \mathbf{R}^{N} \times(0, T),
\end{array}\right.
$$

where $p>1$ and $T>0$, by [19] we have:

(1) Let $u$ be a solution to (1.3) for some $T>0$. Then there exists a unique (nonnegative) Radon measure $\mu$ in $\mathbf{R}^{N}$ as the initial trace of $u$, that is,

$$
\underset{t \rightarrow+0}{\operatorname{ess} \lim _{\mathbf{R}^{N}}} u(x, t) \varphi(x) d x=\int_{\mathbf{R}^{N}} \varphi(x) d \mu(x)
$$

for all $\varphi \in C_{0}\left(\mathbf{R}^{N}\right)$. Furthermore, there exists a positive constant $\gamma_{*}$ depending only on $N$ and $p$ such that

$$
\sup _{x \in \mathbf{R}^{N}} \mu(B(x, \sigma)) \leq \gamma_{*} \sigma^{N-\frac{2}{p-1}}
$$

for $0<\sigma \leq T^{\frac{1}{2}}$. In particular, in the case of $p=1+2 / N$,

$$
\sup _{x \in \mathbf{R}^{N}} \mu(B(x, \sigma)) \leq \gamma_{*}\left[\log \left(e+\frac{T^{\frac{1}{2}}}{\sigma}\right)\right]^{-\frac{N}{2}}
$$

holds for $0<\sigma \leq T^{\frac{1}{2}}$. 
(2) Let $u$ be a solution to (1.3) for some $T>0$. Let $\mu$ be a (nonnegative) Radon measure in $\mathbf{R}^{N}$. Then $\mu$ is the initial trace of $u$ if and only if $u$ is a solution to (1.3) with

$$
u(\cdot, 0)=\mu \quad \text { in } \quad \mathbf{R}^{N} .
$$

See also [2] and [6]. Estimates (1.4) and (1.5) give optimal necessary conditions on the existence of solutions to problem (1.3) with (1.6) (see [19, Theorems 1.3, 1.4 and 1.5]). Compared with semilinear parabolic equation (1.3), much less is known about necessary conditions on the existence of solutions to semilinear parabolic systems and there are no results corresponding to assertions (1) and (2) for parabolic system (1.1) even for the case of $D_{1}=D_{2}$.

In this paper we obtain necessary conditions on the existence of solutions to semilinear parabolic system (1.1) by being divided into the the following six cases (see Figure 1):
(A) $\frac{q+1}{p q-1}<\frac{N}{2}$
(B) $\frac{q+1}{p q-1}=\frac{N}{2}$ and $p<q$;
(D) $\frac{q+1}{p q-1}>\frac{N}{2}$ and $q>1+\frac{2}{N}$;
(F) $\frac{q+1}{p q-1}>\frac{N}{2}$ and $q<1+\frac{2}{N}$.

(C) $\frac{q+1}{p q-1}=\frac{N}{2}$ and $p=q$;

(E) $\frac{q+1}{p q-1}>\frac{N}{2} \quad$ and $\quad q=1+\frac{2}{N}$;
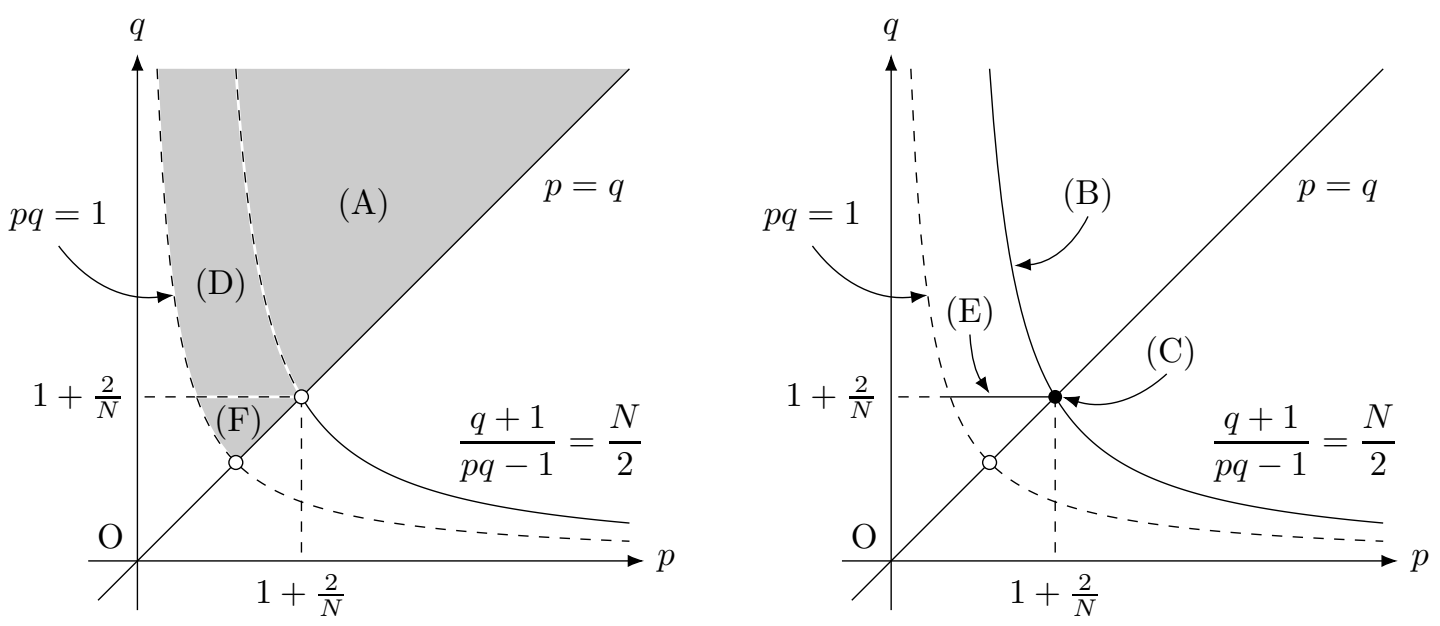

Figure 1

Our necessary conditions in cases $(\mathrm{A}),(\mathrm{C})$ and $(\mathrm{F})$ can be regarded as generalizations of the necessary conditions on the existence of solutions to problem (1.3) in the cases $p>1+2 / N, p=1+2 / N$ and $1<p<1+2 / N$, respectively. On the other hand, our necessary conditions in cases (B), (D) and (E) are specific to parabolic systems. 
We formulate the definition of the solution to (1.1). For any Radon measure $\mu$ in $\mathbf{R}^{N}$, we set

$$
[S(t) \mu](x):=\int_{\mathbf{R}^{N}} G(x-y, t) d \mu(y), \quad \text { where } \quad G(x, t)=(4 \pi t)^{-\frac{N}{2}} \exp \left(-\frac{|x|^{2}}{4 t}\right) .
$$

We also write

$$
[S(t) \mu](x)=\int_{\mathbf{R}^{N}} G(x-y, t) \mu(y) d y
$$

if $\mu$ is a nonnegative measurable function in $\mathbf{R}^{N}$. Then $S(t) \mu$ is the unique solution to the heat equation $\partial_{t} u=\Delta u$ in $\mathbf{R}^{N} \times(0, \infty)$ with $u(0)=\mu$ under a suitable growth condition on the solution at the space infinity.

Definition 1.1 Let $0<T \leq \infty$ and let $(u, v)$ be a pair of nonnegative measurable functions in $\mathbf{R}^{N} \times(0, T)$.

(i) We say that $(u, v)$ is a solution to (1.1) in $\mathbf{R}^{N} \times(0, T)$ if $u(x, t)<\infty, v(x, t)<\infty$ and

$$
\begin{aligned}
& u(x, t)=\left[S\left(D_{1}(t-\tau)\right) u(\tau)\right](x)+\int_{\tau}^{t}\left[S_{1}\left(D_{1}(t-s)\right) v(s)^{p}\right](x) d s, \\
& v(x, t)=\left[S\left(D_{2}(t-\tau)\right) v(\tau)\right](x)+\int_{\tau}^{t}\left[S_{1}\left(D_{2}(t-s)\right) u(s)^{q}\right](x) d s,
\end{aligned}
$$

for almost all $x \in \mathbf{R}^{N}$ and $0<\tau<t<T$.

(ii) Let $\mu$ and $\nu$ be Radon measures in $\mathbf{R}^{N}$. We say that $(u, v)$ is a solution to (1.1) with (1.2) in $\mathbf{R}^{N} \times(0, T)$ if $u(x, t)<\infty, v(x, t)<\infty$ and

$$
\begin{aligned}
& u(x, t)=\left[S\left(D_{1}(t)\right) \mu\right](x)+\int_{0}^{t}\left[S_{1}\left(D_{1}(t-s)\right) v(s)^{p}\right](x) d s, \\
& v(x, t)=\left[S\left(D_{2}(t)\right) \nu\right](x)+\int_{0}^{t}\left[S_{1}\left(D_{2}(t-s)\right) u(s)^{q}\right](x) d s,
\end{aligned}
$$

for almost all $x \in \mathbf{R}^{N}$ and $0<t<T$.

Remark 1.1 Let $(u, v)$ be a solution to (1.1) with (1.2) in $\mathbf{R}^{N} \times(0, T)$ in the sense of Definition 1.1 (ii), where $T>0$. Then it follows from Fubini's theorem that $(u, v)$ is a solution to (1.1) in $\mathbf{R}^{N} \times(0, T)$ in the sense of Definition 1.1 (i).

We are ready to state the main results of this paper. Theorem 1.1 shows the existence and the uniqueness of the initial trace of a solution to (1.1) and gives an upper estimate of the strength of the singularity of the initial trace.

Theorem 1.1 Let $N \geq 1, D_{1}>0, D_{2}>0,0<p \leq q$ with $p q>1$ and $T>0$. Let $(u, v)$ be a solution to (1.1) in $\mathbf{R}^{N} \times(0, T)$. Then there exists a unique pair of Radon measures $(\mu, \nu)$ such that

$$
\begin{aligned}
& \underset{t \rightarrow+0}{\operatorname{ess} \lim _{\mathbf{R}^{N}} u(y, t) \phi(y) d y}=\int_{\mathbf{R}^{N}} \phi(y) d \mu(y), \\
& \underset{t \rightarrow+0}{\operatorname{ess} \lim _{\mathbf{R}^{N}} v(y, t) \phi(y) d y}=\int_{\mathbf{R}^{N}} \phi(y) d \nu(y),
\end{aligned}
$$


for all $\phi \in C_{0}\left(\mathbf{R}^{N}\right)$. Furthermore, there exists a positive constant $\gamma$ depending only on $N$, $D_{1}, D_{2}, p$ and $q$ such that

$$
\sup _{x \in \mathbf{R}^{N}} \mu(B(x, \sigma)) \leq \gamma \sigma^{N-\frac{2(p+1)}{p q-1}}, \quad \sup _{x \in \mathbf{R}^{N}} \nu(B(x, \sigma)) \leq \gamma \sigma^{N-\frac{2(q+1)}{p q-1}},
$$

for $0<\sigma \leq T^{\frac{1}{2}}$

Remark 1.2 In case $(\mathrm{F})$, it follows from $p \leq q$ and $q<1+2 / N$ that

$$
\frac{p+1}{p q-1}-\frac{N}{2}>\frac{p+1}{p q-1}-\frac{1}{q-1}=\frac{(p+1)(q-1)-(p q-1)}{(p q-1)(q-1)}=\frac{q-p}{(p q-1)(q-1)} \geq 0 .
$$

Then we see that the functions

$$
(0, \infty) \ni \sigma \mapsto \sigma^{N-\frac{2(p+1)}{p q-1}} \quad \text { and } \quad(0, \infty) \ni \sigma \mapsto \sigma^{N-\frac{2(q+1)}{p q-1}}
$$

are monotonically decreasing. This implies that estimate (1.9) is equivalent to

$$
\sup _{x \in \mathbf{R}^{N}} \mu\left(B\left(x, T^{\frac{1}{2}}\right)\right) \leq \gamma T^{\frac{N}{2}-\frac{p+1}{p q-1}}, \quad \sup _{x \in \mathbf{R}^{N}} \nu\left(B\left(x, T^{\frac{1}{2}}\right)\right) \leq \gamma T^{\frac{N}{2}-\frac{q+1}{p q-1}} .
$$

Similarly, in cases (D) and (E), the estimates for $\nu$ of (1.9) are equivalent to

$$
\sup _{x \in \mathbf{R}^{N}} \nu\left(B\left(x, T^{\frac{1}{2}}\right)\right) \leq \gamma T^{\frac{N}{2}-\frac{q+1}{p q-1}} .
$$

As an application of Theorem 1.1, we obtain the following result, which corresponds to assertion (2).

Theorem 1.2 Assume the same conditions as in Theorem 1.1. Let $\mu$ and $\nu$ be Radon measures in $\mathbf{R}^{N}$ and $0<T \leq \infty$.

(i) Let $(u, v)$ be a solution to (1.1) with (1.2) in $\mathbf{R}^{N} \times(0, T)$. Then (1.8) holds.

(ii) Let $(u, v)$ be a solution to (1.1) in $\mathbf{R}^{N} \times(0, T)$. Assume (1.8). Then $(u, v)$ is a solution to (1.1) with (1.2) in $\mathbf{R}^{N} \times(0, T)$.

In Theorem 1.3 we improve estimate (1.9) in cases (B), (C), (D) and (E).

Theorem 1.3 Assume the same conditions as in Theorem 1.1. Let $(\mu, \nu)$ be a pair of Radon measures in $\mathbf{R}^{N}$ satisfying (1.8).

(1) Consider case (B). Then there exists $\gamma_{B}>0$ such that

$$
\begin{aligned}
& \sup _{x \in \mathbf{R}^{N}} \int_{0}^{\sigma} \tau^{-1}\left[\frac{\mu(B(x, \tau))}{\tau^{N-\frac{2(p+1)}{p q-1}}}\right]^{q} d \tau+\sup _{x \in \mathbf{R}^{N}} \nu(B(x, \sigma)) \leq \gamma_{B}\left[\log \left(e+\frac{T^{\frac{1}{2}}}{\sigma}\right)\right]^{-\frac{1}{p q-1}} \\
& \text { for } 0<\sigma \leq T^{\frac{1}{2}} .
\end{aligned}
$$


(2) Consider case (C). Then there exists $\gamma_{C}>0$ such that

$$
\sup _{x \in \mathbf{R}^{N}} \mu(B(x, \sigma))+\sup _{x \in \mathbf{R}^{N}} \nu(B(x, \sigma)) \leq \gamma_{C}\left[\log \left(e+\frac{T^{\frac{1}{2}}}{\sigma}\right)\right]^{-\frac{N}{2}}
$$

for $0<\sigma \leq T^{\frac{1}{2}}$

(3) Consider case (D). Then there exists $\gamma_{D}>0$ such that

$$
\sup _{x \in \mathbf{R}^{N}} \int_{0}^{T^{\frac{1}{2}}} \tau^{-1}\left[\frac{\mu(B(x, \tau))}{\tau^{N-\frac{N+2}{q}}}\right]^{q} d \tau+\sup _{x \in \mathbf{R}^{N}} \nu\left(B\left(x, T^{\frac{1}{2}}\right)\right) \leq \gamma_{D} T^{\frac{N}{2}-\frac{q+1}{p q-1}} .
$$

(4) Consider case (E). Then there exists $\gamma_{E}>0$ such that

$$
\sup _{x \in \mathbf{R}^{N}} \int_{0}^{T^{\frac{1}{2}}} \tau^{-1} \mu(B(x, \tau))^{q} d \tau+\sup _{x \in \mathbf{R}^{N}} \nu\left(B\left(x, T^{\frac{1}{2}}\right)\right) \leq \gamma_{E} T^{\frac{N}{2}-\frac{q+1}{p q-1}} .
$$

Here the constants $\gamma_{B}, \gamma_{C}, \gamma_{D}$ and $\gamma_{E}$ depend only on $N, D_{1}, D_{2}, p$ and $q$.

Remark 1.3 As in Remark 1.2, the estimates in assertions (3) and (4) are equivalent to

$$
\begin{aligned}
& \sup _{x \in \mathbf{R}^{N}} \int_{0}^{\sigma} \tau^{-1}\left[\frac{\mu(B(x, \tau))}{\tau^{N-\frac{N+2}{q}}}\right]^{q} d \tau+\sup _{x \in \mathbf{R}^{N}} \nu(B(x, \sigma)) \leq \gamma_{D} \sigma^{N-\frac{2(q+1)}{p q-1}}, \\
& \sup _{x \in \mathbf{R}^{N}} \int_{0}^{\sigma} \tau^{-1} \mu(B(x, \tau))^{q} d \tau+\sup _{x \in \mathbf{R}^{N}} \nu(B(x, \sigma)) \leq \gamma_{E} \sigma^{N-\frac{2(q+1)}{p q-1}},
\end{aligned}
$$

for all $0<\sigma \leq T^{1 / 2}$, respectively.

As corollaries of our theorems, we obtain the following results, which show the validity of estimate (1.9) and Theorem 1.3. Note that Corollary 1.1 has been already proved in [13. for $D_{1}=D_{2}$ and in [15] for $D_{1} \neq D_{2}$.

Corollary 1.1 Let $N \geq 1,0<p \leq q$ with $p q>1$. Assume that

$$
\frac{q+1}{p q-1} \geq \frac{N}{2}
$$

Then problem (1.1) possesses no global-in-time nontrivial solutions.

Remark 1.4 Let $N \geq 1$ and $0<p \leq q$ with $p q>1$. Then (1.10) is an optimal condition for the nonexistence of global-in-time nontrivial solutions to (1.1). Indeed, if

$$
\frac{q+1}{p q-1}<\frac{N}{2}
$$

then problem (1.1) possesses a global-in-time positive solution. See [13] and [15]. 
Corollary 1.2 Let $N \geq 1$ and $0<p \leq q$ with $p q>1$.

(a) Consider case (A). Let $(\mu, \nu)$ be a pair of nonnegative measurable functions in $\mathbf{R}^{N}$ such that

$$
\mu(x) \geq c_{a, 1}|x|^{-\frac{2(p+1)}{p q-1}}, \quad \nu(x) \geq c_{a, 2}|x|^{-\frac{2(q+1)}{p q-1}},
$$

in a neighborhood of 0 , where $c_{a, 1}, c_{a, 2}>0$. Then problem (1.1) with (1.2) possesses no local-in-time solutions if either $c_{a, 1}$ or $c_{a, 2}$ is sufficiently large.

(b) Consider case (B). Let $(\mu, \nu)$ be a pair of nonnegative measurable functions in $\mathbf{R}^{N}$ such that

$$
\mu(x) \geq\left. c_{b, 1}|x|^{-\frac{2(p+1)}{p q-1}}|\log | x\right|^{-\frac{p}{p q-1}}, \quad \nu(x) \geq c_{b, 2}|x|^{-N}|\log | x||^{-\frac{1}{p q-1}-1},
$$

in a neighborhood of 0 , where $c_{b, 1}, c_{b, 2}>0$. Then problem (1.1) with (1.2) possesses no local-in-time solutions if either $c_{b, 1}$ or $c_{b, 2}$ is sufficiently large.

(c) Consider case $(\mathrm{C})$. Let $(\mu, \nu)$ be a pair of nonnegative measurable functions in $\mathbf{R}^{N}$ such that

$$
\mu(x) \geq c_{c, 1}|x|^{-N}|\log | x||^{-\frac{N}{2}-1}, \quad \nu(x) \geq c_{c, 2}|x|^{-N}|\log | x||^{-\frac{N}{2}-1},
$$

in a neighborhood of 0 , where $c_{c, 1}, c_{c, 2}>0$. Then problem (1.1) with (1.2) possesses no local-in-time solutions if either $c_{c, 1}$ or $c_{c, 2}$ is sufficiently large.

(d) Consider case (D). Let $\mu$ be a nonnegative measurable function in $\mathbf{R}^{N}$ such that

$$
\mu(x) \geq|x|^{-\frac{N+2}{q}} h_{1}(|x|)
$$

in a neighborhood of 0 . Here $h_{1}$ is a positive continuous function in $(0,1]$ such that $s^{-\epsilon} h(s)$ is monotonically decreasing in $(0, \delta)$ for some $\epsilon>0$ and $\delta>0$. Let $\nu$ be a Radon measure in $\mathbf{R}^{N}$. Assume either

$$
\int_{0}^{1} \tau^{-1} h_{1}(\tau)^{q} d \tau=\infty \quad \text { or } \quad \sup _{x \in \mathbf{R}^{N}} \nu(B(x, 1))=\infty .
$$

Then problem (1.1) with (1.2) possesses no local-in-time solutions.

(e) Consider case (E). Let $\mu$ be a nonnegative measurable function in $\mathbf{R}^{N}$ such that

$$
\mu(x) \geq|x|^{-N} h_{2}(|x|)
$$

in a neighborhood of 0 , where $h_{2}$ is a positive continuous function in $(0,1]$. Let $\nu$ be a Radon measure in $\mathbf{R}^{N}$. Assume either

$$
\int_{0}^{1}\left[\int_{0}^{r} \tau^{-1} h_{2}(\tau) d \tau\right]^{q} r^{-1} d r=\infty \quad \text { or } \quad \sup _{x \in \mathbf{R}^{N}} \nu(B(x, 1))=\infty .
$$

Then problem (1.1) with (1.2) possesses no local-in-time solutions. 
(f) Consider case $(\mathrm{F})$. Let $(\mu, \nu)$ be a pair of Radon measures in $\mathbf{R}^{N}$. Then problem (1.1) with (1.2) possesses no local-in-time solutions if either

$$
\sup _{x \in \mathbf{R}^{N}} \mu(B(x, 1))=\infty \quad \text { or } \sup _{x \in \mathbf{R}^{N}} \nu(B(x, 1))=\infty .
$$

Corollary 1.2 actually gives optimal conditions for the nonexistence of local-in-time solutions to problem (1.1) with (1.2). Indeed, in case (A), if

$$
0 \leq \mu(x) \leq c_{a, 1}^{\prime}|x|^{-\frac{2(p+1)}{p q-1}} \quad \text { and } \quad 0 \leq \nu(x) \leq c_{a, 1}^{\prime}|x|^{-\frac{2(q+1)}{p q-1}} \quad \text { in } \quad \mathbf{R}^{N}
$$

hold with sufficiently small positive constants $c_{a, 1}^{\prime}$ and $c_{a, 2}^{\prime}$, then problem (1.1) with (1.2) possesses a global-in-time solution (see [22, Corollaries 3.1 and 3.2]). Similar results also hold in the other cases (B), (C), (D), (E) and (F). See a forthcoming paper [17].

The rest of this paper is organized as follows. In Section 2 we recall some properties of the Gauss kernel and prove some preliminary lemmas. In Section 3 we modify the arguments in [13] to prove Theorem [1.1. In Section 4 we follow the arguments in [19] to prove Theorem 1.2. In Sections 5, 6 and 7 we develop the arguments in [19] to prove Theorem 1.3. The proof of Theorem 1.3 requires more delicate arguments than those in [19]. Section 8 is devoted to the proofs of Corollaries 1.1 and 1.2 .

\section{Preliminary lemmas}

In this section we recall some properties of $S(t) \mu$ and prove some preliminary lemmas. Throughout this paper, by the letter $C$ we denote generic positive constants depending only on $N, D_{1}, D_{2}, p$ and $q$, and they may have different values also within the same line.

It follows from (1.7) that

$$
\int_{\mathbf{R}^{N}} G(x, t) d x=1
$$

Then Jensen's inequality implies that

$$
\begin{aligned}
{[S(t) \mu](x) } & =\int_{\mathbf{R}^{N}} G(x-y, t) \mu(y) d y \\
& \leq\left(\int_{\mathbf{R}^{N}} G(x-y, t) \mu(y)^{\alpha} d y\right)^{\frac{1}{\alpha}}=\left[S(t) \mu^{\alpha}\right](x)^{\frac{1}{\alpha}}, \quad x \in \mathbf{R}^{N}, t>0,
\end{aligned}
$$

for any $\alpha \geq 1$. On the other hand, since $G=G(x, t)$ satisfies

$$
G(x, t)=\int_{\mathbf{R}^{N}} G(x-y, t-s) G(y, s) d y, \quad x \in \mathbf{R}^{N}, 0<s<t,
$$

we find

$$
[S(t) \mu](x)=[S(t-s)(S(s) \mu)](x), \quad x \in \mathbf{R}^{N}, 0<s<t .
$$

Furthermore, we have the following lemmas. 
Lemma 2.1 (i) Let $\mu \in L_{\text {loc }}^{1}\left(\mathbf{R}^{N}\right)$ be nonnegative in $\mathbf{R}^{N}$. Then there exists $C>0$ depending only on $N$ such that

$$
\int_{B(0, \rho)} G(x-y, t) \mu(y) d y \geq C G\left(x, \frac{t}{2}\right) \int_{B(0, \rho)} \mu(y) d y
$$

for $x \in \mathbf{R}^{N}, \rho>0$ and $t \geq \rho^{2}$.

(ii) Let $\mu$ be a Radon measure in $\mathbf{R}^{N}$. Then

$$
\int_{B(0, \rho)} G(x-y, t) d \mu(y) \geq C G\left(x, \frac{t}{2}\right) \int_{B(0, \rho)} d \mu(y)
$$

for $x \in \mathbf{R}^{N}, \rho>0$ and $t \geq \rho^{2}$.

Proof. We prove assertion (i). Let $x \in \mathbf{R}^{N}, \rho>0$ and $t \geq \rho^{2}$. Since $|x-y|^{2} \leq 2\left(|x|^{2}+|y|^{2}\right)$, for $y \in B(0, \rho)$, we have

$$
\exp \left(-\frac{|x-y|^{2}}{4 t}\right) \geq \exp \left(-\frac{|x|^{2}}{2 t}\right) \cdot \exp \left(-\frac{|y|^{2}}{2 t}\right) \geq e^{-\frac{1}{2}} \exp \left(-\frac{|x|^{2}}{2 t}\right) .
$$

This implies that

$$
\int_{B(0, \rho)} G(x-y, t) \mu(y) d y \geq 2^{-\frac{N}{2}} e^{-\frac{1}{2}} G\left(x, \frac{t}{2}\right) \int_{B(0, \rho)} \mu(y) d y,
$$

that is, assertion (i) follows. Similarly, assertion (ii) follows. The proof is complete.

Lemma 2.2 Let $\alpha>0, \beta>0$ and $L \geq 0$. Then

$$
\begin{aligned}
& \int_{\mathbf{R}^{N}} G(x-y, t-s) G\left(y, \frac{s+L}{\alpha}\right)^{\beta} d y \\
& \geq\left(\frac{4 \pi}{\alpha}\right)^{-\frac{N}{2}(\beta-1)} \beta^{-\frac{N}{2}}\left(\frac{\min \{\alpha \beta, 1\}}{\max \{\alpha \beta, 1\}}\right)^{\frac{N}{2}}(s+L)^{-\frac{N}{2}(\beta-1)} G\left(x, \frac{t+L}{\max \{\alpha \beta, 1\}}\right)
\end{aligned}
$$

for $x \in \mathbf{R}^{N}$ and $0<s<t$.

Proof. Let $x, y \in \mathbf{R}^{N}$ and $0<s<t$. It follows that

$$
\begin{aligned}
G\left(y, \frac{s+L}{\alpha}\right)^{\beta} & =\left[\frac{4 \pi(s+L)}{\alpha}\right]^{-\frac{N(\beta-1)}{2}}\left[\frac{4 \pi(s+L)}{\alpha \beta}\right]^{-\frac{N}{2}} \beta^{-\frac{N}{2}} \exp \left(-\frac{\alpha \beta|y|^{2}}{4(s+L)}\right) \\
& =\left(\frac{4 \pi}{\alpha}\right)^{-\frac{N(\beta-1)}{2}} \beta^{-\frac{N}{2}}(s+L)^{-\frac{N}{2}(\beta-1)} G\left(y, \frac{s+L}{\alpha \beta}\right) .
\end{aligned}
$$

This together with (2.2) implies that

$$
\begin{aligned}
& \int_{\mathbf{R}^{N}} G(x-y, t-s) G\left(y, \frac{s+L}{\alpha}\right)^{\beta} d y \\
& =\left(\frac{4 \pi}{\alpha}\right)^{-\frac{N(\beta-1)}{2}} \beta^{-\frac{N}{2}}(s+L)^{-\frac{N}{2}(\beta-1)} \int_{\mathbf{R}^{N}} G(x-y, t-s) G\left(y, \frac{s+L}{\alpha \beta}\right) d y \\
& =\left(\frac{4 \pi}{\alpha}\right)^{-\frac{N}{2}(\beta-1)} \beta^{-\frac{N}{2}}(s+L)^{-\frac{N}{2}(\beta-1)} G\left(x, t-s+\frac{s+L}{\alpha \beta}\right) .
\end{aligned}
$$


Since

$$
\frac{t+L}{\max \{1, \alpha \beta\}} \leq t-s+\frac{s+L}{\alpha \beta} \leq \frac{t+L}{\min \{1, \alpha \beta\}},
$$

by (2.3) we obtain the desired inequality, and the proof is complete.

We prove the following basic lemma.

Lemma 2.3 For any $k \geq 1$, there exists $m \in\{1,2, \ldots\}$ with the following property: For any $R>0$ and $z \in \mathbf{R}^{N}$, there exists $\left\{z_{i}\right\}_{i=1}^{m} \subset \mathbf{R}^{N}$ such that

$$
B(z, k R) \subset \bigcup_{i=1}^{m} B\left(z_{i}, R\right) \text {. }
$$

Proof. For any $k \geq 1$, we can find $m \in\{1,2, \ldots\}$ and $\left\{x_{i}\right\}_{i=1}^{m} \subset \mathbf{R}^{N}$ such that

$$
B(0, k) \subset \bigcup_{i=1}^{m} B\left(x_{i}, 1\right) .
$$

Then (2.4) holds with $z_{i}:=z+R x_{i}(i=1, \ldots, m)$, and Lemma 2.3 follows.

As a direct consequence of Lemma 2.3, we have:

Lemma 2.4 Let $\mu$ be a Radon measure in $\mathbf{R}^{N}$ such that

$$
\sup _{x \in \mathbf{R}^{N}} \mu(B(x, R))<\infty
$$

for some $R>0$. Then, for any $k \geq 1$, there exists $m \in\{1,2, \ldots\}$ such that

$$
\sup _{x \in \mathbf{R}^{N}} \mu(B(x, k R)) \leq m \sup _{x \in \mathbf{R}^{N}} \mu(B(x, R))<\infty .
$$

We prepare the Besicovitch covering theorem, which will be used in the proof of Theorem 1.2 , See 14 .

Lemma 2.5 There exists an integer $m$ depending only on $N$ with the following property: If $\mathcal{F}$ is a collection of closed balls in $\mathbf{R}^{N}$ with positive radius such that

$$
\sup \{\operatorname{diam} B: B \in \mathcal{F}\}<\infty
$$

and $A$ is the set of centers of balls in $\mathcal{F}$, then there exist $\mathcal{B}_{1}, \ldots, \mathcal{B}_{m} \subset \mathcal{F}$ such that $\mathcal{B}_{i}$ $(i=1, \ldots, m)$ is a countable collection of disjoint balls in $\mathcal{F}$ and

$$
A \subset \bigcup_{i=1}^{m} \bigcup_{B \in \mathcal{B}_{i}} B .
$$




\section{Proof of Theorem 1.1}

We prove Theorem [1.1. For this aim, it suffices to consider the case of $T=1$ and $D:=\min \left\{D_{1}, D_{2}\right\}=1$. Indeed, let $(u, v)$ be a solution to (1.1) in $\mathbf{R}^{N} \times(0, T)$, where $T>0$. Let $\lambda>0$ be such that $\lambda^{2} \min \left\{D_{1}, D_{2}\right\}=1$. Set

$$
\tilde{u}(x, t):=T^{\frac{p+1}{p q-1}} u\left(\lambda^{-1} T^{\frac{1}{2}} x, T t\right), \quad \tilde{v}(x, t):=T^{\frac{q+1}{p q-1}} u\left(\lambda^{-1} T^{\frac{1}{2}} x, T t\right),
$$

in $\mathbf{R}^{N} \times(0,1)$. Then $(\tilde{u}, \tilde{v})$ satisfies

$$
\left\{\begin{array}{lll}
\partial_{t} \tilde{u}=\tilde{D}_{1} \Delta \tilde{u}+\tilde{v}^{p} & \text { in } & \mathbf{R}^{N} \times(0,1), \\
\partial_{t} \tilde{v}=\tilde{D}_{2} \Delta \tilde{v}+\tilde{u}^{q} & \text { in } & \mathbf{R}^{N} \times(0,1), \\
\tilde{u}, \tilde{v} \geq 0 & \text { in } & \mathbf{R}^{N} \times(0,1),
\end{array}\right.
$$

where $\tilde{D}_{1}=\lambda^{2} D_{1}$ and $\tilde{D}_{2}=\lambda^{2} D_{2}$. Here $\min \left\{\tilde{D}_{1}, \tilde{D}_{2}\right\}=1$.

Let $(u, v)$ be a solution to (1.1) in $\mathbf{R}^{N} \times(0,1)$ and $D=1$. Since

$$
G\left(x, D_{i} t\right)=\left(4 \pi D_{i}\right)^{-\frac{N}{2}} \exp \left(-\frac{|x|^{2}}{4 D_{i} t}\right) \geq\left(D^{\prime}\right)^{-\frac{N}{2}} G(x, t), \quad x \in \mathbf{R}^{N}, t>0,
$$

where $i=1,2$ and $D^{\prime}:=\max \left\{D_{1}, D_{2}\right\}$, by Definition 1.1 (i) we see that

$$
\begin{aligned}
& \infty>\left(D^{\prime}\right)^{\frac{N}{2}} u(x, t) \geq[S(t-\tau) u(\tau)](x)+\int_{\tau}^{t}\left[S(t-s) v(s)^{p}\right](x) d s, \\
& \infty>\left(D^{\prime}\right)^{\frac{N}{2}} v(x, t) \geq[S(t-\tau) v(\tau)](x)+\int_{\tau}^{t}\left[S(t-s) u(s)^{q}\right](x) d s,
\end{aligned}
$$

for almost all $x \in \mathbf{R}^{N}$ and $0<\tau<t<1$. Then we apply the same argument as in the proof of [19, Lemma 2.3] and we can prove the existence and the uniqueness of the initial trace of $(u, v)$. So it remains to prove (1.9) for the proof of Theorem 1.1.

Let

$$
z \in \mathbf{R}^{N}, \quad 0<\rho<1 / \sqrt{5}, \quad 0<\tau \leq \rho^{2}<1 .
$$

Then the functions

$$
U(x, t):=u\left(x+z, t+\tau+2 \rho^{2}\right), \quad V(x, t):=v\left(x+z, t+\tau+2 \rho^{2}\right),
$$

are defined for almost all $x \in \mathbf{R}^{N}$ and $t \in\left[0, t_{*}\right)$, where $t_{*}:=1-\tau-2 \rho^{2} \geq 2 \rho^{2}$. By (3.2) we have

$$
\begin{aligned}
& \infty>\left(D^{\prime}\right)^{\frac{N}{2}} U(x, t) \geq[S(t) U(0)](x)+\int_{0}^{t}\left[S(t-s) V(s)^{p}\right](x) d s, \\
& \infty>\left(D^{\prime}\right)^{\frac{N}{2}} V(x, t) \geq[S(t) V(0)](x)+\int_{0}^{t}\left[S(t-s) U(s)^{q}\right](x) d s,
\end{aligned}
$$


for almost all $x \in \mathbf{R}^{N}, t \in\left[0, t_{*}\right)$ and $\tau \in\left(0, \rho^{2}\right]$. By Lemma 2.1 and (3.2) we see that

$$
\begin{aligned}
U(x, 0) & =u\left(x+z, \tau+2 \rho^{2}\right) \geq\left(D^{\prime}\right)^{-\frac{N}{2}}\left[S\left(2 \rho^{2}\right) u(\tau)\right](x+z) \\
& \geq\left(D^{\prime}\right)^{-\frac{N}{2}} \int_{B(z, \rho)} G\left(x+z-y, 2 \rho^{2}\right) u(y, \tau) d y \geq C M_{z, \tau}(u) G\left(x, \rho^{2}\right), \\
V(x, 0) & =v\left(x+z, \tau+2 \rho^{2}\right) \geq\left(D^{\prime}\right)^{-\frac{N}{2}}\left[S\left(2 \rho^{2}\right) v(\tau)\right](x+z) \\
& \geq\left(D^{\prime}\right)^{-\frac{N}{2}} \int_{B(z, \rho)} G\left(x+z-y, 2 \rho^{2}\right) v(y, \tau) d y \geq C M_{z, \tau}(v) G\left(x, \rho^{2}\right),
\end{aligned}
$$

for almost all $x \in \mathbf{R}^{N}$ and $\tau \in\left(0, \rho^{2}\right]$, where

$$
M_{z, \tau}(u):=\int_{B(z, \rho)} u(x, \tau) d x, \quad M_{z, \tau}(v):=\int_{B(z, \rho)} v(x, \tau) d x
$$

By (3.5) and (3.6) we have

$$
U(x, t) \geq c_{*} M_{z, \tau}(u) G\left(x, t+\rho^{2}\right), \quad V(x, t) \geq c_{*} M_{z, \tau}(v) G\left(x, t+\rho^{2}\right),
$$

for almost all $x \in \mathbf{R}^{N}, t \in\left[0, t_{*}\right)$ and $\tau \in\left(0, \rho^{2}\right]$. Here $c_{*}$ is a positive constant depending only on $N, p, q, D_{1}$ and $D_{2}$.

Lemma 3.1 Let $\rho \in(0,1)$ and $\tau \in(0,1)$ and assume (3.3). Furthermore, assume that there exist positive constants $a, b$ and $c \geq 1$ such that

$$
U(x, t) \geq a t^{b} G\left(x, t+\rho^{2}\right)^{c}
$$

for almost all $x \in \mathbf{R}^{N}$ and $t \in\left[0,2 \rho^{2}\right]$. Then there exists a positive constant $\gamma_{1}$ depending only on $N, D_{1}, D_{2}, p$ and $q$ such that

$$
U(x, t) \geq \gamma_{1} \frac{a^{p q}}{(q b+1)^{p}(p q b+p+1)} t^{p q b+p+1} G\left(x, t+\rho^{2}\right)^{p q c}
$$

for almost all $x \in \mathbf{R}^{N}$ and $t \in\left[0,2 \rho^{2}\right]$.

Proof. We follow the argument in the proof of [13, Lemma 2.4] to prove Lemma 3.1. Since $q>1$, by (2.1), (2.2), (3.5) and (3.8) we have

$$
\begin{aligned}
V(x, t) & \geq\left(D^{\prime}\right)^{-\frac{N}{2}} \int_{0}^{t}\left[S(t-s) U(s)^{q}\right](x) d s \\
& \geq C a^{q} \int_{0}^{t} \int_{\mathbf{R}^{N}} s^{q b} G(x-y, t-s) G\left(y, s+\rho^{2}\right)^{q c} d y d s \\
& \geq C a^{q} \int_{0}^{t} s^{q b}\left(\int_{\mathbf{R}^{N}} G(x-y, t-s) G\left(y, s+\rho^{2}\right) d y\right)^{q c} d s \\
& \geq C \frac{a^{q}}{q b+1} t^{q b+1} G\left(x, t+\rho^{2}\right)^{q c}
\end{aligned}
$$


for almost all $x \in \mathbf{R}^{N}$ and $t \in\left[0,2 \rho^{2}\right]$. Since $p q>1$, repeating the above argument and using (3.9), instead of (3.8), we obtain

$$
\begin{aligned}
U(x, t) & \geq\left(D^{\prime}\right)^{-\frac{N}{2}} \int_{0}^{t}\left[S(t-s) V(s)^{p}\right](x) d s \\
& \geq C \frac{a^{p q}}{(q b+1)^{p}} \int_{0}^{t} \int_{\mathbf{R}^{N}} s^{p(q b+1)} G(x-y, t-s) G\left(y, s+\rho^{2}\right)^{p q c} d y d s \\
& \geq C \frac{a^{p q}}{(q b+1)^{p}} \int_{0}^{t} s^{p(q b+1)}\left(\int_{\mathbf{R}^{N}} G(x-y, t-s) G\left(y, s+\rho^{2}\right) d y\right)^{p q c} d s \\
& \geq C \frac{a^{p q}}{(q b+1)^{p}(p q b+p+1)} t^{p q b+p+1} G\left(x, t+\rho^{2}\right)^{p q c}
\end{aligned}
$$

for almost all $x \in \mathbf{R}^{N}$ and $t \in\left[0,2 \rho^{2}\right]$. Thus Lemma 3.1 follows.

Combining Lemma 3.1 and (3.7), we have:

Lemma 3.2 Let $(u, v)$ satisfy (3.2). Then there exists $C_{*}>0$ such that

$$
\sup _{z \in \mathbf{R}^{N}} \int_{B(z, \rho)} u(y, \tau) d y \leq C_{*} \rho^{N-\frac{2(p+1)}{p q-1}}
$$

for all $\rho \in(0,1 / \sqrt{5})$ and almost all $\tau \in\left(0, \rho^{2}\right]$.

Proof. Let $z \in \mathbf{R}^{N}$ and $\rho \in(0,1 / \sqrt{5})$. Combining Lemma 3.1 and (3.7), we obtain

$$
\infty>U(x, t) \geq a_{n} t^{b_{n}} G\left(x, t+\rho^{2}\right)^{c_{n}}, \quad n=0,1,2, \ldots,
$$

for almost all $x \in \mathbf{R}^{N}, t \in\left[0,2 \rho^{2}\right]$ and $\tau \in\left(0, \rho^{2}\right]$. Here $\left\{a_{n}\right\}_{n=0}^{\infty},\left\{b_{n}\right\}_{n=0}^{\infty}$ and $\left\{c_{n}\right\}_{n=0}^{\infty}$ are sequences defined by

$$
a_{n+1}:=\gamma_{1} \frac{a_{n}^{p q}}{\left(q b_{n}+1\right)^{p}\left(p q b_{n}+p+1\right)}, \quad b_{n+1}:=p q b_{n}+p+1, \quad c_{n+1}:=p q c_{n},
$$

for $n=0,1,2, \ldots$ with

$$
a_{0}:=c_{*} M_{z, \tau}(u), \quad b_{0}:=0, \quad c_{0}:=1 .
$$

Here $c_{*}$ and $\gamma_{1}$ are as in (3.7) and Lemma 3.1, respectively. Then we observe that

$$
b_{n}=\left[(p q)^{n}-1\right] \frac{p+1}{p q-1}, \quad c_{n}=(p q)^{n},
$$

for $n=0,1,2, \ldots$. Furthermore, we can find $C>0$ such that

$$
\log a_{n+1} \geq p q \log a_{n}-C(n+1) .
$$

Then there exists a constant $a_{*}>0$ such that

$$
a_{n} \geq a_{*}^{(p q)^{n}} M_{z, \tau}(u)^{(p q)^{n}}, \quad n=0,1,2, \ldots
$$


(See also [19] and [24, Lemma 2.20 (i)].) Combining (3.10), (3.11) and (3.12), we see that

$$
\begin{aligned}
\infty>U(x, t) & \geq a_{*}^{(p q)^{n}} M_{z, \tau}(u)^{(p q)^{n}} t^{\left[(p q)^{n}-1\right] \frac{p+1}{p q-1}} G\left(x, t+\rho^{2}\right)^{(p q)^{n}} \\
& =\left[a_{*} M_{z, \tau}(u) t^{\frac{p+1}{p q-1}} G\left(x, t+\rho^{2}\right)\right]^{(p q)^{n}} t^{-\frac{p+1}{p q-1}}, \quad n=0,1,2, \ldots,
\end{aligned}
$$

for almost all $x \in \mathbf{R}^{N}, t \in\left[0,2 \rho^{2}\right]$ and $\tau \in\left(0, \rho^{2}\right]$. This implies that

$$
a_{*} M_{z, \tau}(u) t^{\frac{p+1}{p-1}} G\left(x, t+\rho^{2}\right) \leq 1
$$

for almost all $x \in \mathbf{R}^{N}, t \in\left[0,2 \rho^{2}\right]$ and $\tau \in\left(0, \rho^{2}\right]$. Then we deduce that

$$
a_{*} M_{z, \tau}(u)\left(\rho^{2}\right)^{\frac{p+1}{p q-1}}\left(8 \pi \rho^{2}\right)^{-\frac{N}{2}} \leq 1,
$$

that is,

$$
\int_{B(z, \rho)} u(x, \tau) d x=M_{z, \tau}(u) \leq(8 \pi)^{\frac{N}{2}} a_{*}^{-1} \rho^{N-\frac{2(p+1)}{p q-1}}
$$

for almost all $\tau \in\left(0, \rho^{2}\right]$. This completes the proof.

We complete the proof of Theorem 1.1.

Proof of Theorem 1.1. It suffices to prove (1.9) under the assumption that $T=1$ and $D=1$. First, we prove that

$$
\sup _{z \in \mathbf{R}^{N}} \mu(B(z, \sigma)) \leq C \sigma^{N-\frac{2(p+1)}{p q-1}}
$$

for $0<\sigma \leq 1$. By Lemma 3.2 we have

$$
\sup _{z \in \mathbf{R}^{N}} \int_{B(z, \rho)} u(x, \tau) d x \leq C_{*} \rho^{N-\frac{2(p+1)}{p q-1}}
$$

for all $\rho \in(0,1 / \sqrt{5})$ and almost all $\tau \in\left(0, \rho^{2}\right]$, where $C_{*}$ is as in Lemma 3.2. Let $\zeta \in C_{0}\left(\mathbf{R}^{N}\right)$ be such that

$$
0 \leq \zeta \leq 1 \quad \text { in } \quad \mathbf{R}^{N}, \quad \zeta=1 \quad \text { in } \quad B(0, \rho / 2), \quad \zeta=0 \quad \text { outside } B(0, \rho) .
$$

Then, by (1.8) and (3.14) we have

$$
\begin{aligned}
C_{*} \rho^{N-\frac{2(p-1)}{p q-1}} & \geq \int_{B(z, \rho)} u(x, \tau) d x \geq \int_{\mathbf{R}^{N}} u(x, \tau) \zeta(x-z) d x \\
& \rightarrow \int_{\mathbf{R}^{N}} \zeta(x-z) d \mu(x) \geq \mu(B(z, \rho / 2))
\end{aligned}
$$

as $\tau \rightarrow 0$, for $z \in \mathbf{R}^{N}$ and $\rho \in(0,1 / \sqrt{5})$. This together with Lemma 2.4 implies that

$$
\sup _{z \in \mathbf{R}^{N}} \mu(B(z, 2 \sqrt{5} \rho)) \leq C \sup _{z \in \mathbf{R}^{N}} \mu(B(z, \rho / 2)) \leq C \rho^{N-\frac{2(p+1)}{p q-1}}
$$


for $\rho \in(0,1 / \sqrt{5})$. Then we set $\sigma:=2 \sqrt{5} \rho$ and obtain (3.13).

Next we prove that

$$
\sup _{z \in \mathbf{R}^{N}} \nu(B(z, \sigma)) \leq C \sigma^{N-\frac{2(q+1)}{p q-1}}
$$

for $0<\sigma \leq 1$. It follows from Lemma 3.2 with $\tau=r^{2}$ that

$$
\sup _{z \in \mathbf{R}^{N}} \int_{B(z, r)} u\left(x, r^{2}\right) d x \leq C_{*} r^{N-\frac{2(p+1)}{p q-1}}
$$

for almost all $r \in(0,1 / \sqrt{5})$. Let $z \in \mathbf{R}^{N}$. Since Lemma 2.2 implies that

$$
\int_{\mathbf{R}^{N}} G(x-y, t-s) G\left(y, s+\rho^{2}\right)^{p} d y \geq C\left(s+\rho^{2}\right)^{-\frac{N}{2}(p-1)} G\left(x, \frac{t+\rho^{2}}{\max \{p, 1\}}\right)
$$

for $x \in \mathbf{R}^{N}, \rho>0$ and $\rho^{2}<s<t$, by (3.4), (3.5) and (3.7) we have

$$
\begin{aligned}
u\left(x, \tau+4 \rho^{2}\right) & =U\left(x-z, 2 \rho^{2}\right) \\
& \geq\left(D^{\prime}\right)^{-\frac{N}{2}} \int_{0}^{2 \rho^{2}} \int_{\mathbf{R}^{N}} G\left(x-z-y, 2 \rho^{2}-s\right) V(y, s)^{p} d y d s \\
& \geq C M_{z, \tau}(v)^{p} \int_{\rho^{2}}^{2 \rho^{2}} \int_{\mathbf{R}^{N}} G\left(x-z-y, 2 \rho^{2}-s\right) G\left(y, s+\rho^{2}\right)^{p} d y d s \\
& \geq C M_{z, \tau}(v)^{p} G\left(x-z, \frac{3 \rho^{2}}{\max \{p, 1\}}\right) \int_{\rho^{2}}^{2 \rho^{2}}\left(s+\rho^{2}\right)^{-\frac{N}{2}(p-1)} d s \\
& \geq C M_{z, \tau}(v)^{p} \rho^{-N(p-1)+2} G\left(x-z, \frac{3 \rho^{2}}{\max \{p, 1\}}\right)
\end{aligned}
$$

for almost all $x \in \mathbf{R}^{N}, \rho \in(0,1 / 5)$ and $\tau \in\left(0, \rho^{2}\right]$. Since $\tau+4 \rho^{2} \leq 5 \rho^{2}<1 / 5$ for $\rho \in(0,1 / 5)$, by (3.16) we obtain

$$
\begin{aligned}
C_{*}\left(\tau+4 \rho^{2}\right)^{\frac{N}{2}-\frac{p+1}{p q-1}} & \geq \int_{B\left(z, \sqrt{\tau+4 \rho^{2}}\right)} u\left(x, \tau+4 \rho^{2}\right) d x \geq \int_{B(z, 2 \rho)} u\left(x, \tau+4 \rho^{2}\right) d x \\
& \geq C M_{z, \tau}(v)^{p} \rho^{-N(p-1)+2} \int_{B(0, \rho)} G\left(x, \frac{3 \rho^{2}}{\max \{p, 1\}}\right) d x \\
& \geq C M_{z, \tau}(v)^{p} \rho^{-N(p-1)+2}
\end{aligned}
$$

for almost all $\rho \in(0,1 / 5)$ and $\tau \in\left(0, \rho^{2}\right]$. This implies that

$$
\sup _{z \in \mathbf{R}^{N}} \int_{B(z, \rho)} v(x, \tau) d x=\sup _{z \in \mathbf{R}^{N}} M_{z, \tau}(v) \leq C \rho^{N-\frac{2(q+1)}{p q-1}}
$$

for all $\rho \in(0,1 / 5)$ and almost all $\tau \in\left(0, \rho^{2}\right]$. Then we apply the same argument as in the proof of (3.13) to obtain (3.15). Therefore, by (3.13) and (3.15) we have (1.9), and the proof of Theorem 1.1 is complete. 


\section{Proof of Theorem 1.2}

Applying similar arguments as in the proof of [19, Theorem 1.2], we prove Theorem 1.2, Proof of Theorem 1.2. Similarly to the proof of Theorem 1.1, it suffices to consider the case of $T=1$. We prove assertion (i). By Theorem 1.1 we can find a pair of Radon measures $\left(\mu^{\prime}, \nu^{\prime}\right)$ satisfying

$$
\begin{aligned}
& \underset{t \rightarrow+0}{\operatorname{ess} \lim _{\mathbf{R}^{N}} u(y, t) \eta(y) d y}=\int_{\mathbf{R}^{N}} \eta(y) d \mu^{\prime}(y), \\
& \underset{t \rightarrow+0}{\operatorname{ess} \lim _{\mathbf{R}^{N}} v(y, t) \eta(y) d y}=\int_{\mathbf{R}^{N}} \eta(y) d \nu^{\prime}(y),
\end{aligned}
$$

for all $\eta \in C_{0}\left(\mathbf{R}^{N}\right)$. Furthermore, for any $r>0$, by Lemmas 2.4 and 3.2 we see that

$$
\sup _{z \in \mathbf{R}^{N}} \int_{B(z, r)} u(y, \tau) d y \text { is uniformly bounded for almost all } \tau \in(0,1 / 5) .
$$

Let $\zeta \in C_{0}\left(\mathbf{R}^{N}\right)$ be such that $\zeta \geq 0$ in $\mathbf{R}^{N}$. Let $R>0$ be such that $\operatorname{supp} \zeta \subset B(0, R)$. It follows from Definition 1.1 and Remark 1.1 that

$$
\begin{aligned}
\int_{\mathbf{R}^{N}} u(x, t) \zeta(x) d x & =\int_{\mathbf{R}^{N}} \int_{\mathbf{R}^{N}} G\left(x-y, D_{1}(t-\tau)\right) u(y, \tau) \zeta(x) d x d y \\
& +\int_{\tau}^{t} \int_{\mathbf{R}^{N}} \int_{\mathbf{R}^{N}} G\left(x-y, D_{1}(t-s)\right) v(y, s)^{p} \zeta(x) d x d y d s
\end{aligned}
$$

for almost all $0<\tau<t<T$. Set $\zeta(x, t):=\left[S\left(D_{1} t\right) \zeta\right](x)$. Then

$$
\lim _{t \rightarrow+0}\|\zeta(t)-\zeta\|_{L^{\infty}\left(\mathbf{R}^{N}\right)}=0 .
$$

By (4.1) and (4.2) we have

$$
\begin{gathered}
\int_{\mathbf{R}^{N}} \int_{\mathbf{R}^{N}} G\left(x-y, D_{1}(t-\tau)\right) u(y, \tau) \zeta(x) d x d y \geq \int_{B(0, R)} u(y, \tau) \zeta(y, t-\tau) d y \\
\quad \geq \int_{B(0, R)} u(y, \tau) \zeta(y) d y-C\|\zeta(t-\tau)-\zeta\|_{L^{\infty}(B(0, R))} \\
\rightarrow \int_{\mathbf{R}^{N}} \zeta(y) d \mu^{\prime}(y)-C\|\zeta(t)-\zeta\|_{L^{\infty}(B(0, R)) \quad \text { as } \quad \tau \rightarrow+0 .}
\end{gathered}
$$

This together with (4.3) implies that

$$
\begin{aligned}
\int_{\mathbf{R}^{N}} u(x, t) \zeta(x) d x & \geq \int_{\mathbf{R}^{N}} \zeta(y) d \mu^{\prime}(y)-C\|\zeta(t)-\zeta\|_{L^{\infty}(B(0, R))} \\
& +\int_{0}^{t} \int_{\mathbf{R}^{N}} \int_{\mathbf{R}^{N}} G\left(x-y, D_{1}(t-s)\right) v(y, s)^{p} \zeta(x) d x d y d s
\end{aligned}
$$

for almost all $t \in(0, T)$. By (4.1), (4.4) and (4.5), letting $t \rightarrow+0$, we obtain

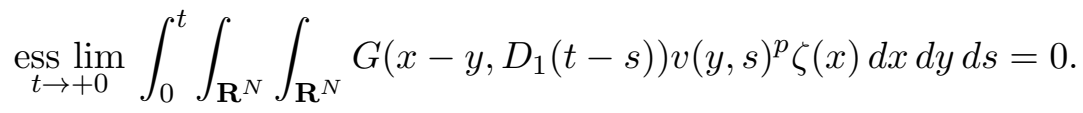


On the other hand, it follows from Definition 1.1 (ii) that

$$
\begin{aligned}
\int_{\mathbf{R}^{N}} u(x, t) \zeta(x) d x & =\int_{\mathbf{R}^{N}} \int_{\mathbf{R}^{N}} G\left(x-y, D_{1} t\right) \zeta(x) d \mu(y) d x \\
& +\int_{0}^{t} \int_{\mathbf{R}^{N}} \int_{\mathbf{R}^{N}} G\left(x, y, D_{1}(t-s)\right) v(y, s)^{p} \zeta(x) d x d y d s
\end{aligned}
$$

for almost all $0<t<T$. Then, by (4.1), (4.4), (4.6) and (4.7) we obtain

$$
\int_{\mathbf{R}^{N}} \zeta(x) d \mu^{\prime}(x)=\underset{t \rightarrow+0}{\operatorname{ess}} \lim _{\mathbf{R}^{N}} \zeta(y, t) d \mu(y)=\int_{\mathbf{R}^{N}} \zeta(y) d \mu(y) .
$$

Since $\zeta$ is arbitrary, we deduce that $\mu=\mu^{\prime}$ in $\mathbf{R}^{N}$. Similarly, we see that $\nu=\nu^{\prime}$ in $\mathbf{R}^{N}$. Then assertion (i) follows.

We prove assertion (ii). Let $(u, v)$ be a solution to (1.1) in $\mathbf{R}^{N} \times(0,1)$. Let $0<t<1$ and $n=1,2, \ldots$ By the Besicovitch covering lemma we can find an integer $m$ depending only on $N$ and a set $\left\{x_{k, i}\right\}_{k=1, \ldots, m, i \in \mathbf{N}} \subset \mathbf{R}^{N} \backslash B\left(0, n t^{1 / 2}\right)$ such that

$$
B_{k, i} \cap B_{k, j}=\emptyset \quad \text { if } i \neq j \quad \text { and } \quad \mathbf{R}^{N} \backslash B\left(0, n t^{\frac{1}{2}}\right) \subset \bigcup_{k=1}^{m} \bigcup_{i=1}^{\infty} B_{k, i},
$$

where $B_{k, i}:=\overline{B\left(x_{k, i}, t^{1 / 2}\right)}$. By Lemma 2.4, Lemma 3.2 and (4.8) we obtain

$$
\begin{aligned}
& \underset{0<\tau<t / 5}{\operatorname{ess} \sup _{0<}} \int_{\mathbf{R}^{N} \backslash B\left(0, n t^{\frac{1}{2}}\right)} G\left(y, D_{1}(t-\tau)\right) u(y, \tau) d y \\
& \leq \sum_{k=1}^{m} \sum_{i=1}^{\infty} \operatorname{ess}_{\substack{0<\tau<t / 5 \\
0<\tau<}} \int_{B_{k, i}} G\left(y, D_{1}(t-\tau)\right) u(y, \tau) d y
\end{aligned}
$$

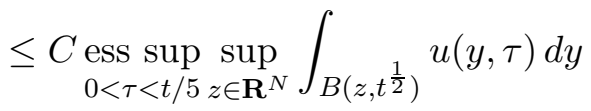

$$
\begin{aligned}
& \times \sum_{k=1}^{m} \sum_{i=1}^{\infty} \sup _{0<\tau<t / 5} \sup _{y \in B_{k, i}}(t-\tau)^{-\frac{N}{2}} \exp \left(-\frac{|y|^{2}}{4 D_{1}(t-\tau)}\right) \\
& \leq C t^{-\frac{p+1}{p q-1}} \sum_{k=1}^{m} \sum_{i=1}^{\infty} \sup _{y \in B_{k, i}} \exp \left(-\frac{|y|^{2}}{4 D_{1} t}\right) \text {. }
\end{aligned}
$$

On the other hand, since

$$
(n-1) t^{\frac{1}{2}} \leq|z| \leq\left|z-x_{k, i}\right|+\left|x_{k, i}-y\right|+|y| \leq|y|+2 t^{\frac{1}{2}}
$$

for $y, z \in B_{k, i}$, we have

$$
\inf _{y \in B_{k, i}} \frac{|y|^{2}}{t} \geq \frac{|| z\left|-2 t^{\frac{1}{2}}\right|^{2}}{t} \geq \frac{|z|^{2}}{2 t}-C
$$

for $z \in B_{k, i}$ and sufficiently large $n$. Then

$$
\sup _{y \in B_{k, i}} \exp \left(-\frac{|y|^{2}}{4 D_{1} t}\right) \leq C \frac{1}{\left|B_{k, i}\right|} \int_{B_{k, i}} \exp \left(-\frac{|z|^{2}}{8 D_{1} t}\right) d z
$$


for sufficiently large $n$, where $\left|B_{k, i}\right|$ denotes the measure of the ball $B_{k, i}$. This together with (4.8) and (4.9) implies that

$$
\begin{aligned}
& \operatorname{ess~sup}_{0<\tau<t / 5} \int_{\mathbf{R}^{N} \backslash B\left(0, n t^{\frac{1}{2}}\right)} G\left(y, D_{1}(t-\tau)\right) u(y, \tau) d y \\
& \leq C t^{-\frac{N}{2}-\frac{p+1}{p q-1}} \sum_{k=1}^{m} \sum_{i=1}^{\infty} \int_{B_{k, i}} \exp \left(-\frac{|z|^{2}}{8 D_{1} t}\right) d z \\
& \leq C t^{-\frac{N}{2}-\frac{p+1}{p q-1}} \int_{\mathbf{R}^{N} \backslash B\left(0,(n-1) t^{\frac{1}{2}}\right)} \exp \left(-\frac{|z|^{2}}{8 D_{1} t}\right) d z \\
& \leq C t^{-\frac{p+1}{p q-1}} \int_{\mathbf{R}^{N} \backslash B(0, n-1)} \exp \left(-\frac{|z|^{2}}{8 D_{1}}\right) d z \rightarrow 0
\end{aligned}
$$

as $n \rightarrow \infty$. Similarly, by Theorem 1.1 we have

$$
\begin{aligned}
& \int_{\mathbf{R}^{N} \backslash B\left(0, n t^{\frac{1}{2}}\right)} G\left(y, D_{1} t\right) d \mu(y) \\
& \leq C \sup _{z \in \mathbf{R}^{N}} \mu\left(B\left(z, t^{\frac{1}{2}}\right)\right) \sum_{k=1}^{m} \sum_{i=1}^{\infty} \sup _{y \in B_{k, i}} t^{-\frac{N}{2}} \exp \left(-\frac{|y|^{2}}{4 D_{1} t}\right) \\
& \leq C t^{-\frac{p+1}{p q-1}} \int_{\mathbf{R}^{N} \backslash B(0, n-1)} \exp \left(-\frac{|z|^{2}}{8 D_{1}}\right) d z \rightarrow 0
\end{aligned}
$$

as $n \rightarrow \infty$. In particular, by (4.2), (4.10) and (4.11) we see that

$$
\int_{\mathbf{R}^{N}} G\left(y, D_{1}(t-\tau)\right) u(y, \tau) d y<\infty, \quad \int_{\mathbf{R}^{N}} G\left(y, D_{1} t\right) d \mu(y)<\infty,
$$

for all $0<t<1$ and almost all $\tau \in(0, t / 5)$.

Let $\eta_{n} \in C_{0}\left(\mathbf{R}^{N}\right)$ be such that

$$
0 \leq \eta_{n} \leq 1 \quad \text { in } \quad \mathbf{R}^{N}, \quad \eta_{n}=1 \quad \text { on } \quad B\left(0, n t^{\frac{1}{2}}\right), \quad \eta_{n}=0 \quad \text { outside } B\left(0,2 n t^{\frac{1}{2}}\right) .
$$

It follows from (4.12) that

$$
\begin{aligned}
& \left|\int_{\mathbf{R}^{N}} G\left(y, D_{1}(t-\tau)\right) u(y, \tau) d y-\int_{\mathbf{R}^{N}} G\left(y, D_{1} t\right) d \mu(y)\right| \\
& \leq\left|\int_{\mathbf{R}^{N}} G\left(y, D_{1} t\right) u(y, \tau) \eta_{n}(y) d y-\int_{\mathbf{R}^{N}} G\left(y, D_{1} t\right) \eta_{n}(y) d \mu(y)\right| \\
& \quad+\left|\int_{\mathbf{R}^{N}}\left[G\left(y, D_{1}(t-\tau)\right)-G\left(y, D_{1} t\right)\right] u(y, \tau) \eta_{n}(y) d y\right| \\
& \quad+\int_{\mathbf{R}^{N} \backslash B\left(0, n t^{\frac{1}{2}}\right)} G\left(y, D_{1}(t-\tau)\right) u(y, \tau) d y+\int_{\mathbf{R}^{N} \backslash B\left(0, n t^{\frac{1}{2}}\right)} G\left(y, D_{1} t\right) d \mu(y)
\end{aligned}
$$

for $n=1,2, \ldots$ and almost all $\tau \in(0, t / 2)$. By (1.8) we see that

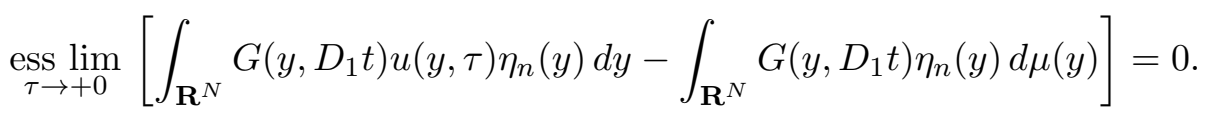


Furthermore, by (4.2) we have

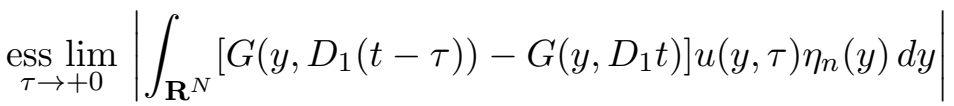

$$
\begin{aligned}
& \leq \sup _{y \in B\left(0,2 n t^{\frac{1}{2}}\right), s \in(4 t / 5, t)}\left|\partial_{t} G\left(y, D_{1} s\right)\right| \operatorname{ess} \limsup _{\tau \rightarrow+0}\left[\tau \int_{B\left(0,2 n t^{\frac{1}{2}}\right)} u(y, \tau) d y\right]=0 .
\end{aligned}
$$

By (4.13), (4.14) and (4.15) we obtain

$$
\begin{aligned}
& \underset{\tau \rightarrow+0}{\operatorname{ess} \lim _{\tau \rightarrow \mathbf{R}^{N}}}\left|\int_{\mathbf{R}^{N}} G\left(y, D_{1}(t-\tau)\right) u(y, \tau) d y-\int_{\mathbf{R}^{N}} G\left(y, D_{1} t\right) d \mu(y)\right| \\
& \leq \operatorname{ess} \sup _{0<\tau<t / 5} \int_{\mathbf{R}^{N} \backslash B\left(0, n t^{\frac{1}{2}}\right)} G\left(y, D_{1}(t-\tau)\right) u(y, \tau) d y+\int_{\mathbf{R}^{N} \backslash B\left(0, n t^{\frac{1}{2}}\right)} G\left(y, D_{1} t\right) d \mu(y)
\end{aligned}
$$

for $n=1,2, \ldots$. This together with (4.10) and (4.11) implies that

$$
\underset{\tau \rightarrow+0}{\operatorname{ess} \lim _{\tau}}\left|\int_{\mathbf{R}^{N}} G\left(y, D_{1}(t-\tau)\right) u(y, \tau) d y-\int_{\mathbf{R}^{N}} G\left(y, D_{1} t\right) d \mu(y)\right|=0 .
$$

Similarly, we see that

$$
\underset{\tau \rightarrow+0}{\operatorname{ess} \lim _{\tau}}\left|\int_{\mathbf{R}^{N}} G\left(y, D_{2}(t-\tau)\right) v(y, \tau) d y-\int_{\mathbf{R}^{N}} G\left(y, D_{2} t\right) d \nu(y)\right|=0 .
$$

These together with Definition 1.1(i) imply that $(u, v)$ is a solution to (1.1) with (1.2) in $\mathbf{R}^{N} \times(0,1)$. Thus assertion (ii) follows, and the proof of Theorem 1.2 is complete.

\section{$5 \quad$ Initial traces in case (B)}

In this section we focus on case (B), that is,

$$
0<p<q, \quad p q>1, \quad \frac{q+1}{p q-1}=\frac{N}{2},
$$

and prove assertion (1) of Theorem [1.3. It follows from (5.1) that

$$
-\frac{N}{2}(p-1)+1>0>-\frac{N}{2}(q-1)+1 .
$$

Similarly to Section 2 , it suffices to consider the case where $T=1$ and $D=1$.

Let

$$
z \in \mathbf{R}^{N}, \quad 0<\rho<1 / \sqrt{10}, \quad 0<\tau \leq \rho^{2}<1 .
$$

Similarly to (3.4), we define $U=U(x, t)$ and $V=V(x, t)$ for $x \in \mathbf{R}^{N}$ and $0<t<t_{*}$, where

$$
t_{*}:=1-\tau-2 \rho^{2}>\frac{1}{2}>2 \rho^{2} .
$$

Then $U$ and $V$ satisfy (3.5) and (3.7) for almost all $x \in \mathbf{R}^{N}, t \in\left(0, t_{*}\right)$ and $\tau \in\left(0, \rho^{2}\right]$. Furthermore, the following lemma holds. 
Lemma 5.1 Assume (5.1) and (5.3). Let $(U, V)$ satisfy (3.5). Assume that there exist constants $a>0, b \geq 1$ and $c \geq 0$ such that

$$
V(x, t) \geq a G\left(x, \frac{t+\rho^{2}}{b}\right)\left[\log \frac{t}{\rho^{2}}\right]^{c}
$$

for almost all $x \in \mathbf{R}^{N}$ and $t \in\left[2 \rho^{2}, t_{*}\right)$, where $\rho>0$. Then there exists a positive constant $\gamma_{2}$ depending only on $N, D_{1}, D_{2}, p$ and $q$ such that

$$
V(x, t) \geq \gamma_{2} a^{p q} b^{\frac{N}{2} q(p-2)+\frac{N}{2}(q-2)}(p c+1)^{-q}(p q c+1)^{-1} G\left(x, \frac{t+\rho^{2}}{q \max \{p b, 1\}}\right)\left[\log \frac{t}{\rho^{2}}\right]^{p q c+1}
$$

for almost all $x \in \mathbf{R}^{N}$ and $t \in\left[2 \rho^{2}, t_{*}\right)$.

For the proof of Lemma [5.1, we prove:

Lemma 5.2 Let $a>-1, b>0$ and $\rho>0$. Then

$$
\int_{\rho^{2}}^{t}\left(s+\rho^{2}\right)^{a}\left[\log \frac{s}{\rho^{2}}\right]^{b} d s \geq \frac{\left(t+\rho^{2}\right)^{a+1}}{4(a+b+2)}\left[\log \frac{t}{\rho^{2}}\right]^{b} \quad \text { for } t \geq 2 \rho^{2} .
$$

Proof. Let $t \geq 2 \rho^{2}$. Set

$$
F(t):=\int_{\rho^{2}}^{t}\left(s+\rho^{2}\right)^{a}\left[\log \frac{s}{\rho^{2}}\right]^{b} d s-\frac{1}{4(a+b+2)}\left(t+\rho^{2}\right)^{a+1}\left[\log \frac{t}{\rho^{2}}\right]^{b} .
$$

Then, since

$$
\begin{aligned}
\int_{1}^{2}(\sigma+1)^{a}(\log \sigma)^{b} d \sigma & =\int_{1}^{2}(\sigma+1)^{a+1} \cdot \frac{\sigma}{\sigma+1} \cdot \sigma^{-1}(\log \sigma)^{b} d \sigma \\
& \geq \frac{1}{2} \cdot 2^{a+1} \int_{1}^{2} \sigma^{-1}(\log \sigma)^{b} d \sigma=\frac{1}{2} \cdot 2^{a+1} \cdot \frac{1}{b+1}(\log 2)^{b+1} \\
& \geq(2 \log 2) \cdot \frac{1}{4(a+b+2)} 2^{a+1}(\log 2)^{b} \geq \frac{1}{4(a+b+2)} 2^{a+1}(\log 2)^{b},
\end{aligned}
$$

we have

$$
\begin{aligned}
F\left(2 \rho^{2}\right) & =\int_{\rho^{2}}^{2 \rho^{2}}\left(s+\rho^{2}\right)^{a}\left[\log \frac{s}{\rho^{2}}\right]^{b} d s-\frac{1}{4(a+b+2)}\left(2 \rho^{2}\right)^{a+1}(\log 2)^{b} \\
& =\left(\rho^{2}\right)^{a+1}\left\{\int_{1}^{2}(\sigma+1)^{a}(\log \sigma)^{b} d \sigma-\frac{1}{4(a+b+2)} 2^{a+1}(\log 2)^{b}\right\} \geq 0 .
\end{aligned}
$$

Since

$$
\begin{aligned}
F^{\prime}(t)=(t & \left.+\rho^{2}\right)^{a}\left[\log \frac{t}{\rho^{2}}\right]^{b} \\
& -\frac{1}{4(a+b+2)}\left\{(a+1)\left(t+\rho^{2}\right)^{a}\left[\log \frac{t}{\rho^{2}}\right]^{b}+b\left(t+\rho^{2}\right)^{a} \cdot \frac{t+\rho^{2}}{t}\left[\log \frac{t}{\rho^{2}}\right]^{b-1}\right\}
\end{aligned}
$$




$$
\begin{aligned}
& =\left\{1-\frac{a+1}{4(a+b+2)}-\frac{b}{4(a+b+2)} \cdot \frac{t+\rho^{2}}{t}\left[\log \frac{t}{\rho^{2}}\right]^{-1}\right\}\left(t+\rho^{2}\right)^{a}\left[\log \frac{t}{\rho^{2}}\right]^{b} \\
& \geq\left\{\frac{3}{4}-\frac{1}{4} \cdot \frac{3}{2}(\log 2)^{-1}\right\}\left(t+\rho^{2}\right)^{a}\left[\log \frac{t}{\rho^{2}}\right]^{b} \geq 0
\end{aligned}
$$

for $t \geq 2 \rho^{2}$, by (5.6) we have $F(t) \geq 0$ for $t \geq 2 \rho^{2}$. Thus Lemma 5.2 follows.

Proof of Lemma 5.1. By Lemma 2.2, Lemma 5.2, (3.5), (5.2) and (5.5) we have

$$
\begin{aligned}
U(x, t) & \geq\left(D^{\prime}\right)^{-\frac{N}{2}} \int_{\rho^{2}}^{t} \int_{\mathbf{R}^{N}} G(x-y, t-s) V(y, s)^{p} d y d s \\
& \geq C a^{p} \int_{\rho^{2}}^{t} \int_{\mathbf{R}^{N}}\left[\log \frac{s}{\rho^{2}}\right]^{p c} G(x-y, t-s) G\left(y, \frac{s+\rho^{2}}{b}\right)^{p} d y d s \\
& \geq C a^{p} b^{\frac{N}{2}(p-1)}\left(\frac{\min \{p b, 1\}}{\max \{p b, 1\}}\right)^{\frac{N}{2}} G\left(x, \frac{t+\rho^{2}}{\max \{p b, 1\}}\right) \\
& \times \int_{\rho^{2}}^{t}\left(s+\rho^{2}\right)^{-\frac{N}{2}(p-1)}\left[\log \frac{s}{\rho^{2}}\right]^{p c} d s \\
& \geq C \frac{a^{p} b^{\frac{N}{2}(p-2)}}{p c+1}\left(t+\rho^{2}\right)^{-\frac{N}{2}(p-1)+1} G\left(x, \frac{t+\rho^{2}}{\max \{p b, 1\}}\right)\left[\log \frac{t}{\rho^{2}}\right]^{p c}
\end{aligned}
$$

for almost all $x \in \mathbf{R}^{N}$ and $t \in\left[2 \rho^{2}, t_{*}\right)$. Since

$$
-\frac{N}{2}(p q-q)+q-\frac{N}{2}(q-1)=-\frac{N}{2}(p q-1)+q=-\frac{q+1}{p q-1}(p q-1)+q=-1,
$$

by Lemma 2.2 we obtain

$$
\begin{aligned}
& V(x, t) \geq\left(D^{\prime}\right)^{-\frac{N}{2}} \int_{\rho^{2}}^{t} \int_{\mathbf{R}^{N}} G(x-y, t-s) U(y, s)^{q} d y d s \\
& \geq\left(D^{\prime}\right)^{-\frac{N}{2}}\left(C \frac{a^{p} b^{\frac{N}{2}(p-2)}}{p c+1}\right)^{q} \int_{\rho^{2}}^{t}\left(s+\rho^{2}\right)^{-\frac{N}{2}(p q-q)+q}\left[\log \frac{s}{\rho^{2}}\right]^{p q c} \\
& \times\left[\int_{\mathbf{R}^{N}} G(x-y, t-s) G\left(x, \frac{s+\rho^{2}}{\max \{p b, 1\}}\right)^{q} d y\right] d s \\
& \geq C\left(\frac{a^{p} b^{\frac{N}{2}(p-2)}}{p c+1}\right)^{q}[\max \{p b, 1\}]^{\frac{N}{2}(q-2)} G\left(x, \frac{t+\rho^{2}}{q \max \{p b, 1\}}\right) \\
& \geq\left.C \frac{a^{p q} b^{\frac{N}{2} q(p-2)+\frac{N}{2}(q-2)}\left(s+\rho^{2}\right)^{-\frac{N}{2}(p q-q)+q-\frac{N}{2}(q-1)}}{(p c+1)^{q}}\left[\log \frac{s}{\rho^{2}}\right]^{p q c} d s, \frac{t+\rho^{2}}{q \max \{p b, 1\}}\right) \int_{\rho^{2}}^{t}\left(s+\rho^{2}\right)^{-1}\left[\log \frac{s}{\rho^{2}}\right]^{p q c} d s \\
& \geq C \frac{a^{p q} b^{\frac{N}{2} q(p-2)+\frac{N}{2}(q-2)}}{(p c+1)^{q}(p q c+1)} G\left(x, \frac{t+\rho^{2}}{q \max \{p b, 1\}}\right)\left[\log \frac{t}{\rho^{2}}\right]^{p q c+1}
\end{aligned}
$$

for almost all $x \in \mathbf{R}^{N}$ and $t \in\left[2 \rho^{2}, t_{*}\right)$. Thus Lemma 5.1 follows. 
Combining Lemma 5.1 and (3.7), we have:

Lemma 5.3 Assume (5.1). Let $(u, v)$ satisfy (3.2). Then there exists a constant $C_{*}>0$ such that

$$
\sup _{z \in \mathbf{R}^{N}} \int_{B(z, \rho)} v(x, \tau) d x \leq C_{*}\left[\log \left(e+\frac{1}{\rho}\right)\right]^{-\frac{1}{p q-1}}
$$

for all $\rho \in(0,1 / \sqrt{10})$ and almost all $\tau \in\left(0, \rho^{2}\right]$.

Proof. Let $z \in \mathbf{R}^{N}$ and $\rho \in(0,1 / \sqrt{10})$. Combining Lemma 5.1 and (3.7), we obtain

$$
\infty>V(x, t) \geq a_{n} G\left(x, \frac{t+\rho^{2}}{b_{n}}\right)\left[\log \frac{t}{\rho^{2}}\right]^{c_{n}}, \quad n=0,1,2, \ldots,
$$

for almost all $x \in \mathbf{R}^{N}, t \in\left[2 \rho^{2}, t_{*}\right)$ and $\tau \in\left(0, \rho^{2}\right]$. Here $\left\{a_{n}\right\}_{n=0}^{\infty},\left\{b_{n}\right\}_{n=0}^{\infty}$ and $\left\{c_{n}\right\}_{n=0}^{\infty}$ are sequences defined by

$$
a_{n+1}:=\gamma_{2} \frac{a_{n}^{p q} b_{n}^{\frac{N}{2}} q(p-2)+\frac{N}{2}(q-2)}{\left(p c_{n}+1\right)^{q}\left(p q c_{n}+1\right)}, \quad b_{n+1}:=q \max \left\{p b_{n}, 1\right\}, \quad c_{n+1}:=p q c_{n}+1,
$$

for $n=0,1,2, \ldots$ with

$$
a_{0}:=c_{*} M_{z, \tau}(v), \quad b_{0}=1, \quad c_{0}=0,
$$

where $\gamma_{2}$ is as in Lemma 5.1. Since $p b_{1}=p q \max \{p, 1\}>1$, we have

$$
b_{n}=(p q)^{n-1} b_{1}, \quad c_{n}=\frac{(p q)^{n}-1}{p q-1},
$$

for $n=1,2,3, \ldots$ Furthermore, similarly to (3.12), we can find $a_{*}>0$ such that

$$
a_{n} \geq a_{*}^{(p q)^{n}} M_{z, \tau}(v)^{(p q)^{n}}, \quad n=1,2, \ldots
$$

By (5.8), (5.9) and (5.10) we see that

$$
\begin{aligned}
\infty>V(x, t) & \geq a_{*}^{(p q)^{n}} M_{z, \tau}(v)^{(p q)^{n}} G\left(x, \frac{t+\rho^{2}}{(p q)^{n-1} b_{1}}\right)\left[\log \frac{t}{\rho^{2}}\right]^{\frac{(p q)^{n}-1}{p q-1}} \\
& \geq\left\{a_{*} M_{z, \tau}(v)\left[\log \frac{t}{\rho^{2}}\right]^{\frac{1}{p q-1}}\right\}^{(p q)^{n}} G\left(x, \frac{t+\rho^{2}}{(p q)^{n-1} b_{1}}\right)\left[\log \frac{t}{\rho^{2}}\right]^{\frac{-1}{p q-1}}
\end{aligned}
$$

for almost all $x \in \mathbf{R}^{N}, t \in\left[2 \rho^{2}, t_{*}\right)$ and $\tau \in\left(0, \rho^{2}\right]$ and for all $n=1,2, \ldots$ This implies that

$$
a_{*} M_{z, \tau}(v)\left[\log \frac{t}{\rho^{2}}\right]^{\frac{1}{p q-1}} \leq 1
$$

for almost all $t \in\left[2 \rho^{2}, t_{*}\right)$ and $\tau \in\left(0, \rho^{2}\right]$. Since $t_{*}>1 / 2$, we deduce that

$$
\int_{B(z, \rho)} v(x, \tau) d x=M_{z, \tau}(v) \leq a_{*}^{-1}\left[\log \frac{1}{2 \rho^{2}}\right]^{-\frac{1}{p q-1}} \leq C\left[\log \left(e+\frac{1}{\rho}\right)\right]^{-\frac{1}{p q-1}}
$$


for almost all $\tau \in\left(0, \rho^{2}\right]$. This implies (5.7), and the proof is complete.

Proof of assertion (1) of Theorem 1.3. Applying the same argument as in the proof of Theorem 1.1, by Lemma 5.3 we observe that

$$
\sup _{z \in \mathbf{R}^{N}} \nu(B(z, \sigma)) \leq C\left[\log \left(e+\frac{1}{\sigma}\right)\right]^{-\frac{1}{p q-1}}
$$

for $0<\sigma \leq 1$. It remains to prove

$$
\sup _{z \in \mathbf{R}^{N}} \int_{0}^{\sigma} \tau^{-1}\left[\frac{\mu(B(z, \tau))}{\tau^{N-\frac{2(p+1)}{p q-1}}}\right]^{q} d \tau \leq C\left[\log \left(e+\frac{1}{\sigma}\right)\right]^{-\frac{1}{p q-1}}
$$

for $0<\sigma \leq 1$.

Let $\sigma_{*}=1 / \sqrt{10}$ and $0<\sigma<\sigma_{*}$. Applying Lemma 5.3 with $\tau=\sigma^{2}<1 / 10$, we have

$$
\sup _{z \in \mathbf{R}^{N}} \int_{B(z, \sigma)} v\left(x, \sigma^{2}\right) d x \leq C\left[\log \left(e+\frac{1}{\sigma}\right)\right]^{-\frac{1}{p q-1}}
$$

for almost all $\sigma \in\left(0, \sigma_{*}\right)$. This together with Lemma 2.4 implies that

$$
\sup _{z \in \mathbf{R}^{N}} \int_{B(z, \sqrt{2} \sigma)} v\left(x, \sigma^{2}\right) d x \leq C\left[\log \left(e+\frac{1}{\sigma}\right)\right]^{-\frac{1}{p q-1}}
$$

for almost all $\sigma \in\left(0, \sigma_{*}\right)$. On the other hand, by Theorems 1.1 and 1.2 we see that $(u, v)$ is a solution to problem (1.1) with (1.2) in $\mathbf{R}^{N} \times(0,1)$. Then it follows from Definition 1.1(ii) and (3.1) that

$$
\begin{aligned}
u(x, t) & \geq \int_{\mathbf{R}^{N}} G\left(x-y, D_{1} t\right) d \mu(y) \\
& \geq\left(D^{\prime}\right)^{-\frac{N}{2}} \int_{\mathbf{R}^{N}} G(x-y, t) d \mu(y)=\left(D^{\prime}\right)^{-\frac{N}{2}}[S(t) \mu](x)
\end{aligned}
$$

for almost all $x \in \mathbf{R}^{N}$ and $t \in(0,1)$. Using Definition 1.1 (ii) again, by (3.1) and (5.13) we have

$$
\begin{aligned}
v(x, t) & \geq \int_{0}^{t} \int_{\mathbf{R}^{N}} G\left(x-y, D_{2}(t-s)\right) u(y, s)^{q} d y d s \\
& \geq\left(D^{\prime}\right)^{-\frac{N}{2}(q+1)} \int_{0}^{t} \int_{\mathbf{R}^{N}} G(x-y, t-s)[S(s) \mu](y)^{q} d y d s
\end{aligned}
$$

for almost all $x \in \mathbf{R}^{N}$ and $t \in(0,1)$. Then we see that

$$
\begin{aligned}
& \int_{B(z, \sqrt{2} \sigma)} v\left(x, \sigma^{2}\right) d x \\
& \geq C \int_{B(z, \sqrt{2} \sigma)} \int_{0}^{\sigma^{2}} \int_{\mathbf{R}^{N}} G\left(x-y, \sigma^{2}-s\right)[S(s) \mu](y)^{q} d y d s d x \\
& \geq C \int_{B(z, \sqrt{2} \sigma)} \int_{0}^{\sigma^{2}} \int_{B(z, \sqrt{s})} G\left(x-y, \sigma^{2}-s\right)[S(s) \mu](y)^{q} d y d s d x \\
& =C \int_{0}^{\sigma^{2}} \int_{B(z, \sqrt{s})} \int_{B(z, \sqrt{2} \sigma)} G\left(x-y, \sigma^{2}-s\right)[S(s) \mu](y)^{q} d x d y d s
\end{aligned}
$$


for all $z \in \mathbf{R}^{N}$ and almost all $\sigma \in\left(0, \sigma_{*}\right)$. On the other hand, since $\sqrt{s}+\sqrt{\sigma^{2}-s} \leq \sqrt{2} \sigma$ for $s \in\left(0, \sigma^{2}\right)$, it follows that

$$
B\left(y, \sqrt{\sigma^{2}-s}\right) \subset B(z, \sqrt{2} \sigma) \text { for } y \in B(z, \sqrt{s}) \text { and } s \in\left(0, \sigma^{2}\right) .
$$

This together with (5.14) implies that

$$
\begin{aligned}
& \int_{B(z, \sqrt{2} \sigma)} v\left(x, \sigma^{2}\right) d x \\
& \geq \int_{0}^{\sigma^{2}} \int_{B(z, \sqrt{s})} \int_{B\left(y, \sqrt{\sigma^{2}-s}\right)} G\left(x-y, \sigma^{2}-s\right)[S(s) \mu](y)^{q} d x d y d s \\
& \geq C \int_{0}^{\sigma^{2}} \int_{B(z, \sqrt{s})}[S(s) \mu](y)^{q} d y d s
\end{aligned}
$$

for all $z \in \mathbf{R}^{N}$ and almost all $\sigma \in\left(0, \sigma_{*}\right)$. Furthermore, by Lemma 2.1 we have

$$
[S(s) \mu](y) \geq \int_{B(z, \sqrt{s})} G(y-\xi, s) d \mu(\xi) \geq C G\left(y-z, \frac{s}{2}\right) \mu(B(z, \sqrt{s}))
$$

for $y, z \in \mathbf{R}^{N}$ and $s>0$. Combining (5.12), (5.15) and (5.16), we obtain

$$
\begin{aligned}
{\left[\log \left(e+\frac{1}{\sigma}\right)\right]^{-\frac{1}{p q-1}} } & \geq C \int_{0}^{\sigma^{2}} \mu(B(z, \sqrt{s}))^{q} \int_{B(z, \sqrt{s})} G\left(y-z, \frac{s}{2}\right)^{q} d y d s \\
& \geq C \int_{0}^{\sigma^{2}} \mu(B(z, \sqrt{s}))^{q} s^{-\frac{N}{2}(q-1)} d s \\
& \geq C \int_{0}^{\sigma} \mu(B(z, \tau))^{q} \tau^{-N q+N+1} d \tau
\end{aligned}
$$

for all $z \in \mathbf{R}^{N}$ and $\sigma \in\left(0, \sigma_{*}\right)$. By (5.1) we see that

$$
\begin{aligned}
& (-N q+N+1)-\left\{-q\left(N-\frac{2(p+1)}{p q-1}\right)-1\right\} \\
& =N+2-2 \cdot \frac{p q+q}{p q-1}=N+2-2 \cdot \frac{(p q-1)+(q+1)}{p q-1}=N-2 \cdot \frac{q+1}{p q-1}=0,
\end{aligned}
$$

which together with (5.17) implies that

$$
\sup _{z \in \mathbf{R}^{N}} \int_{0}^{\sigma} \tau^{-1}\left[\frac{\mu(B(z, \tau))}{\tau^{N-\frac{2(p+1)}{p q-1}}}\right]^{q} d \tau \leq C\left[\log \left(e+\frac{1}{\sigma}\right)\right]^{-\frac{1}{p q-1}}
$$

for $0<\sigma<\sigma_{*}=1 / \sqrt{10}$. In the case of $\sigma_{*} \leq \sigma<1$, by (5.18) we see that

$$
\begin{aligned}
\sup _{z \in \mathbf{R}^{N}} \int_{0}^{\sigma} \tau^{-1}\left[\frac{\mu(B(z, \tau))}{\left.\tau^{N-\frac{2(p+1)}{p q-1}}\right]^{q} d \tau}\right. & \leq C+\sup _{z \in \mathbf{R}^{N}} \int_{\sigma_{*}}^{\sigma} \tau^{-1}\left[\frac{\mu(B(z, \tau))}{\tau^{N-\frac{2(p+1)}{p q-1}}}\right]^{q} d \tau \\
& \leq C+C \sup _{z \in \mathbf{R}^{N}} \int_{\sigma_{*} / \sqrt{10}}^{\sigma / \sqrt{10}} \tau^{-1}\left[\frac{\mu(B(z, \sqrt{10} \tau))}{\tau^{N-\frac{2(p+1)}{p q-1}}}\right]^{q} d \tau
\end{aligned}
$$


On the other hand, by Lemma 2.3, for any $z \in \mathbf{R}^{N}$, we can find $\left\{z_{i}\right\}_{i=1}^{m} \subset \mathbf{R}^{N}$ such that

$$
\mu(B(z, \sqrt{10} \tau)) \leq \sum_{i=1}^{m} \mu\left(B\left(z_{i}, \tau\right)\right) \quad \text { for } \tau \in(0,1) .
$$

Here $m$ is independent of $z$ and $\tau$. Then

$$
\mu(B(z, \sqrt{10} \tau))^{q} \leq C \sum_{i=1}^{m} \mu\left(B\left(z_{i}, \tau\right)\right)^{q} \quad \text { for } \tau \in(0,1),
$$

which together with (5.18) implies that

$$
\int_{\sigma_{*} / \sqrt{10}}^{\sigma / \sqrt{10}} \tau^{-1}\left[\frac{\mu(B(z, \sqrt{10} \tau))}{\tau^{N-\frac{2(p+1)}{p q-1}}}\right]^{q} d \tau \leq C \sum_{i=1}^{m} \int_{0}^{\sigma / \sqrt{10}} \tau^{-1}\left[\frac{\mu\left(B\left(z_{i}, \tau\right)\right)}{\tau^{N-\frac{2(p+1)}{p q-1}}}\right]^{q} d \tau \leq C
$$

for all $z \in \mathbf{R}^{N}$. We deduce from (5.19) and (5.20) that

$$
\sup _{z \in \mathbf{R}^{N}} \int_{0}^{\sigma} \tau^{-1}\left[\frac{\mu(B(z, \tau))}{\tau^{N-\frac{2(p+1)}{p q-1}}}\right]^{q} d \tau \leq C \leq C\left[\log \left(e+\frac{1}{\sigma}\right)\right]^{-\frac{1}{p q-1}}
$$

for $\sigma_{*} \leq \sigma<1$. Combining (5.18) and (5.21), we obtain (5.11). Thus assertion (1) of Theorem 1.3 follows.

\section{$6 \quad$ Initial traces in case $(\mathrm{C})$}

In this section we focus on case $(\mathrm{C})$, that is,

$$
\frac{q+1}{p q-1}=\frac{N}{2}, \quad p=q
$$

and prove assertion (2) of Theorem 1.3. It follows from (6.1) that

$$
p=q=1+\frac{2}{N} .
$$

Similarly to Section 3, it suffices to consider the case where $T=1$ and $D=1$. Assume (5.3). Then $(U, V)$ is defined for almost all $x \in \mathbf{R}^{N}, t \in\left(0, t_{*}\right)$ and $\tau \in\left(0, \rho^{2}\right]$, where $t_{*}$ is as in (5.4). Furthermore, $(U, V)$ satisfies (3.5) and (3.7) for almost all $x \in \mathbf{R}^{N}, t \in\left(0, t_{*}\right)$ and $\tau \in\left(0, \rho^{2}\right]$.

Set $W(x, t):=U(x, t)+V(x, t)$ and $M_{z, \tau}(w):=M_{z, \tau}(u)+M_{z, \tau}(v)$. Then we have:

Lemma 6.1 Assume (5.3) and (6.1). Let $(U, V)$ satisfy (3.5). Assume that there exist constants $a>0, b \geq 1$ and $c \geq 0$ such that

$$
W(x, t) \geq a G\left(x, \frac{t+\rho^{2}}{b}\right)\left[\log \frac{t}{\rho^{2}}\right]^{c}
$$


for almost all $x \in \mathbf{R}^{N}$ and $t \in\left[\rho^{2}, t_{*}\right)$. Then there exists a positive constant $\gamma_{3}$ depending only on $N, D_{1}, D_{2}, p$ and $q$ such that

$$
W(x, t) \geq \gamma_{3} \frac{a^{p} b^{\frac{N}{2}(p-2)}}{p c+1} G\left(x, \frac{t+\rho^{2}}{p b}\right)\left[\log \frac{t}{\rho^{2}}\right]^{p c+1}
$$

for almost all $x \in \mathbf{R}^{N}$ and $t \in\left[\rho^{2}, t_{*}\right)$.

Proof. Since $p>1$, it follows that $W(x, t)^{p} \leq C\left[U(x, t)^{p}+V(x, t)^{p}\right]$ for almost all $x \in \mathbf{R}^{N}$ and $t \in\left(0, t_{*}\right)$. Then, by (3.5) and (6.3) we have

$$
\begin{aligned}
W(x, t) & \geq\left(D^{\prime}\right)^{-\frac{N}{2}} \int_{0}^{t} \int_{\mathbf{R}^{N}} G(x-y, t-s)\left[U(y, s)^{p}+V(y, s)^{p}\right] d y d s \\
& \geq C \int_{\rho^{2}}^{t} \int_{\mathbf{R}^{N}} G(x-y, t-s) W(y, s)^{p} d y d s \\
& \geq C a^{p} \int_{\rho^{2}}^{t} \int_{\mathbf{R}^{N}}\left[\log \frac{s}{\rho^{2}}\right]^{p c} G(x-y, t-s) G\left(y, \frac{s+\rho^{2}}{b}\right)^{p} d y d s
\end{aligned}
$$

for almost all $x \in \mathbf{R}^{N}$ and $t \in\left[\rho^{2}, t_{*}\right)$. This together with Lemma 2.2 and (6.2) implies that

$$
\begin{aligned}
W(x, t) & \geq C a^{p} b^{\frac{N}{2}(p-2)} G\left(x, \frac{t+\rho^{2}}{p b}\right) \int_{\rho^{2}}^{t}\left(s+\rho^{2}\right)^{-1}\left[\log \frac{s}{\rho^{2}}\right]^{p c} d s \\
& \geq C a^{p} b^{\frac{N}{2}(p-2)}(p c+1)^{-1} G\left(x, \frac{t+\rho^{2}}{p b}\right)\left[\log \frac{t}{\rho^{2}}\right]^{p c+1}
\end{aligned}
$$

for almost all $x \in \mathbf{R}^{N}$ and $t \in\left[\rho^{2}, t_{*}\right)$. Thus Lemma 6.1 follows.

Proof of assertion (2) of Theorem 1.3. It suffices to prove assertion (2) under the assumption that $T=1$ and $D=1$. Let $z \in \mathbf{R}^{N}, \rho \in(0,1 / \sqrt{10})$ and $\tau \in\left(0, \rho^{2}\right]$. By (3.7) we have

$$
W(x, t) \geq c_{*} M_{z, \tau}(w) G\left(x, t+\rho^{2}\right)
$$

for almost all $x \in \mathbf{R}^{N}, t \in\left[0, t_{*}\right)$ and $\tau \in\left(0, \rho^{2}\right]$. Then, by Lemma 6.1 we obtain

$$
\infty>W(x, t) \geq a_{n} G\left(x, \frac{t+\rho^{2}}{b_{n}}\right)\left[\log \frac{t}{\rho^{2}}\right]^{c_{n}}, \quad n=0,1,2, \ldots,
$$

for almost all $x \in \mathbf{R}^{N}, t \in\left[\rho^{2}, t_{*}\right)$ and $\tau \in\left(0, \rho^{2}\right]$. Here $\left\{a_{n}\right\}_{n=0}^{\infty},\left\{b_{n}\right\}_{n=0}^{\infty}$ and $\left\{c_{n}\right\}_{n=0}^{\infty}$ are sequences defined by

$$
a_{n+1}:=\gamma_{3} \frac{a_{n}^{p} b_{n}^{\frac{N}{2}(p-2)}}{p c_{n}+1}, \quad b_{n+1}:=p b_{n}, \quad c_{n+1}:=p c_{n}+1,
$$

for $n=0,1,2, \ldots$ with

$$
a_{0}:=c_{*} M_{z, \tau}(w), \quad b_{0}:=1, \quad c_{0}:=0,
$$


where $\gamma_{3}$ is as in Lemma 6.1. Then we have

$$
b_{n}=p^{n}, \quad c_{n}=\frac{p^{n}-1}{p-1},
$$

for $n=0,1,2, \ldots$ Furthermore, similarly to (3.12), by (6.5) we can find $a_{*}>0$ such that

$$
a_{n} \geq a_{*}^{p^{n}} M_{z, \tau}(w)^{p^{n}}
$$

for $n=0,1,2, \ldots$ By (6.4), (6.6) and (6.7) we see that

$$
\begin{aligned}
\infty>W(x, t) & \geq a_{*}^{p^{n}} M_{z, \tau}(w)^{p^{n}} G\left(x, \frac{t+\rho^{2}}{p^{n}}\right)\left[\log \frac{t}{\rho^{2}}\right]^{\frac{p^{n}-1}{p-1}} \\
& =\left\{a_{*} M_{z, \tau}(w)\left[\log \frac{t}{\rho^{2}}\right]^{\frac{1}{p-1}}\right\}^{p^{n}} G\left(x, \frac{t+\rho^{2}}{p^{n}}\right)\left[\log \frac{t}{\rho^{2}}\right]^{-\frac{1}{p-1}}
\end{aligned}
$$

for almost all $x \in \mathbf{R}^{N}, t \in\left[\rho^{2}, t_{*}\right)$ and $\tau \in\left(0, \rho^{2}\right]$ and for all $n=0,1,2, \ldots$ This implies that

$$
a_{*} M_{z, \tau}(w)\left[\log \frac{t}{\rho^{2}}\right]^{\frac{1}{p-1}} \leq 1
$$

for almost all $t \in\left[\rho^{2}, t_{*}\right)$ and $\tau \in\left(0, \rho^{2}\right]$. Since $t_{*}>1 / 2$ (see (5.4) $)$, we deduce that

$$
M_{z, \tau}(u)+M_{z, \tau}(v) \leq a_{*}^{-1}\left[\log \frac{1}{2 \rho^{2}}\right]^{-\frac{N}{2}} \leq C\left[\log \left(e+\frac{1}{\rho}\right)\right]^{-\frac{N}{2}}
$$

for all $z \in \mathbf{R}^{N}$ and $\rho \in(0,1 / \sqrt{10})$ and for almost all $\tau \in\left(0, \rho^{2}\right]$. Then, applying the same argument as in the proof of Theorem 1.1, we obtain the desired result, and the proof of assertion (2) of Theorem 1.3 is complete.

\section{Initial traces in cases (D) and (E)}

In this section we focus on cases (D) and (E), that is,

$$
0<p<q, \quad p q>1, \quad \frac{q+1}{p q-1}>\frac{N}{2}, \quad q \geq 1+\frac{2}{N},
$$

and prove assertions (3) and (4) of Theorem 1.3 .

Proof of assertions (3) and (4) of Theorem 1.3. Similarly to Section 3, it suffices to consider the case where $T=1$ and $D=1$. Furthermore, by Theorem [1.1 (see (1.9)) we have only to prove

$$
\sup _{z \in \mathbf{R}^{N}} \int_{0}^{1} \tau^{-1}\left[\frac{\mu(B(z, \tau))}{\tau^{N-\frac{N+2}{q}}}\right]^{q} d \tau \leq \gamma
$$

where $\gamma$ is a positive constant depending only on $N, D_{1}, D_{2}, p$ and $q$. 
Let $\rho_{*}=1 / 5$. By (3.17) with $\tau=\rho^{2}$ we have

$$
\sup _{z \in \mathbf{R}^{N}} \int_{B(z, \rho)} v\left(x, \rho^{2}\right) d x \leq \gamma^{\prime}
$$

for almost all $\rho \in\left(\rho_{*} / 2, \rho_{*}\right)$. Here $\gamma^{\prime}$ is a positive constant depending only on $N, D_{1}, D_{2}$, $p$ and $q$. Then Lemma 2.4 implies that

$$
\sup _{z \in \mathbf{R}^{N}} \int_{B(z, \sqrt{2} \rho)} v\left(x, \rho^{2}\right) d x \leq C \gamma^{\prime}
$$

for almost all $\rho \in\left(\rho_{*} / 2, \rho_{*}\right)$. Furthermore, similarly to (5.14) and (5.15), we have

$$
\begin{aligned}
& \int_{B(z, \sqrt{2} \rho)} v\left(x, \rho^{2}\right) d x \\
& \geq C \int_{0}^{\rho^{2}} \int_{B(z, \sqrt{s})} \int_{B(z, \sqrt{2} \rho)} G\left(x-y, \rho^{2}-s\right)[S(s) \mu](y)^{q} d x d y d s \\
& \geq C \int_{0}^{\rho^{2}} \int_{B(z, \sqrt{s})}[S(s) \mu](y)^{q} d y d s
\end{aligned}
$$

for all $z \in \mathbf{R}^{N}$ and almost all $\rho \in\left(\rho_{*} / 2, \rho_{*}\right)$. Combining (7.2) and (7.3) and applying a similar argument as in (5.16) and (5.17), we obtain

$$
\begin{aligned}
C \gamma^{\prime} & \geq \sup _{z \in \mathbf{R}^{N}} \int_{0}^{\rho^{2}} \int_{B(z, \sqrt{s})}[S(s) \mu](y)^{q} d y d s \\
& \geq C \sup _{z \in \mathbf{R}^{N}} \int_{0}^{\rho^{2}} \mu(B(z, \sqrt{s}))^{q} \int_{B(z, \sqrt{s})} G\left(y-z, \frac{s}{2}\right)^{q} d y d s \\
& \geq C \sup _{z \in \mathbf{R}^{N}} \int_{0}^{\rho^{2}} \mu(B(z, \tau))^{q} \tau^{-N q+N+1} d \tau=C \sup _{z \in \mathbf{R}^{N}} \int_{0}^{\rho^{2}} \tau^{-1}\left[\frac{\mu(B(z, \tau))}{\tau^{N-\frac{N+2}{q}}}\right]^{q} d \tau
\end{aligned}
$$

for all $\rho \in\left(\rho_{*} / 2, \rho_{*}\right)$. This implies that

$$
\sup _{z \in \mathbf{R}^{N}} \int_{0}^{\rho_{*}^{2}} \tau^{-1}\left[\frac{\mu(B(z, \tau))}{\tau^{N-\frac{N+2}{q}}}\right]^{q} d \tau \leq C .
$$

Then, applying a similar argument as in (5.21), we obtain (7.1). Thus assertions (3) and (4) of Theorem 1.3 follows. Therefore the proof of Theorem 1.3 is complete.

\section{Proofs of Corollaries 1.1 and 1.2}

Proof of Corollary 1.1. The proof is by contradiction. Assume that problem (1.1) possesses a global-in-time nontrivial solution. Then, by Definition 1.1 we see that problem (1.1) possesses a global-in-time positive solution $(u, v)$.

Let $\tau>0$ and set $\tilde{u}(x, t):=u(x, t+\tau)$ and $\tilde{v}(x, t):=v(v, t+\tau)$. Then, by Definition 1.1, for almost all $\tau>0$, we see that $(\tilde{u}, \tilde{v})$ is a global-in-time positive solution to (1.1) with 
$(\tilde{u}(0), \tilde{v}(0))=(u(\tau), v(\tau))$. Furthermore, we observe from Theorem 1.2 that $(u(\tau), v(\tau))$ is the initial trace of the solution $(\tilde{u}, \tilde{v})$. Therefore, by Theorem 1.1 we can find $\gamma>0$ such that

$$
\int_{B(0, \sigma)} \tilde{u}(x, 0) d x \leq \gamma \sigma^{N-\frac{2(p+1)}{p q-1}}, \quad \int_{B(0, \sigma)} \tilde{v}(x, 0) d x \leq \gamma \sigma^{N-\frac{2(q+1)}{p q-1}}
$$

for all $\sigma>0$.

Assume that

$$
\frac{q+1}{p q-1}>\frac{N}{2}
$$

Then it follows from (8.1) that

$$
\lim _{\sigma \rightarrow \infty} \int_{B(0, \sigma)} v(x, \tau) d x=\lim _{\sigma \rightarrow \infty} \int_{B(0, \sigma)} \tilde{v}(x, 0) d x=0 .
$$

Since $\tau$ is arbitrary, we see that $v(x, t)=0$ for almost all $x \in \mathbf{R}^{N}$ and $t \in(0, \infty)$. This contradicts that $(u, v)$ is a positive solution. Thus problem (1.1) possesses no global-intime nontrivial solutions under assumption (8.2).

It remains to consider the case of

$$
\frac{q+1}{p q-1}=\frac{N}{2}
$$

We consider case (B). Then, by Theorem 1.3 we can find $\gamma^{\prime}>0$ such that

$$
\int_{B(0, \sigma)} \tilde{v}(x, 0) d \tau \leq \gamma^{\prime}\left[\log \left(e+\frac{T^{\frac{1}{2}}}{\sigma}\right)\right]^{-\frac{1}{p q-1}}
$$

for all $0<\sigma<T^{\frac{1}{2}}$ and all $T>0$. Let $T>1$ and set $\sigma=T^{\frac{1}{4}}$. Then we see that

$$
\int_{B\left(0, T^{\frac{1}{4}}\right)} v(x, \tau) d x=\int_{B\left(0, T^{\frac{1}{4}}\right)} \tilde{v}(x, 0) d x \leq \gamma^{\prime}\left[\log \left(e+T^{\frac{1}{4}}\right)\right]^{-\frac{1}{p q-1}} \rightarrow 0
$$

as $T \rightarrow \infty$. Similarly to the case of (8.2), we deduce that $v(x, t)=0$ for almost all $x \in \mathbf{R}^{N}$ and $t \in(0, \infty)$, which is a contradiction. Case $(\mathrm{C})$ can be treated in the same manner. Therefore problem (1.1) possesses no global-in-time nontrivial solutions under assumption (8.3). Thus Corollary 1.1 follows.

Proof of Corollary 1.2. The proof is by contradiction. Assume that problem (1.1) with (1.2) possesses a solution $(u, v)$ in $\mathbf{R}^{N} \times(0, T)$ for some $T>0$. Theorem 1.2 implies that the initial trace of $(u, v)$ coincides with the initial data of $(u, v)$.

Case (A): It follows from (1.11) that

$$
\int_{B(0, \sigma)} \mu(x) d x \geq C c_{a, 1} \sigma^{N-\frac{2(p+1)}{p q-1}}, \quad \int_{B(0, \sigma)} \nu(x) d x \geq C c_{a, 2} \sigma^{N-\frac{2(q+1)}{p q-1}},
$$

for all sufficiently small $\sigma>0$. This implies that (1.9) does not hold if either $c_{a, 1}$ or $c_{a, 2}$ is sufficiently large. This contradicts Theorem 1.1. Thus assertion (a) follows.

Case (B): It follows from (1.12) that

$$
\int_{B(0, \sigma)} \mu(x) d x \geq C c_{b, 1} \sigma^{N-\frac{2(p+1)}{p q-1}}|\log \sigma|^{-\frac{p}{p q-1}}, \quad \int_{B(0, \sigma)} \nu(x) d x \geq C c_{b, 2}|\log \sigma|^{-\frac{1}{p q-1}},
$$


for all sufficiently small $\sigma>0$. Then

$$
\int_{0}^{\sigma} \tau^{-1}\left[\frac{\mu(B(0, \tau))}{\tau^{N-\frac{2(p+1)}{p q-1}}}\right]^{q} d \tau \geq C^{q} c_{b, 1}^{q} \int_{0}^{\sigma} \tau^{-1}|\log \tau|^{-1-\frac{1}{p q-1}} d \tau \geq C c_{b, 1}^{q}|\log \sigma|^{-\frac{1}{p q-1}}
$$

for all sufficiently small $\sigma>0$. Then these estimates contradict assertion (1) of Theorem 1.3 if either $c_{b, 1}$ or $c_{b, 2}$ is sufficiently large. Thus assertion (b) follows.

Case (C): It follows from (1.13) that

$$
\int_{B(0, \sigma)} \mu(x) d x \geq C c_{c, 1}|\log \sigma|^{-\frac{N}{2}}, \quad \int_{B(0, \sigma)} \nu(x) d x \geq C c_{c, 2}|\log \sigma|^{-\frac{N}{2}},
$$

for all sufficiently small $\sigma>0$. Then these estimates contradict assertion (2) of Theorem 1.3 if either $c_{c, 1}$ or $c_{c, 2}$ is sufficiently large. Thus assertion (c) follows.

Case (D): Let $\epsilon>0$ be as in assertion (d). It follows from $q>1+2 / N$ that $(N+2) / q<N$. Then, by (1.14) we have

$$
\begin{aligned}
\int_{B(0, \sigma)} \mu(x) d x & \geq C \int_{0}^{\sigma} r^{-\frac{N+2}{q}+N-1+\epsilon} r^{-\epsilon} h_{1}(r) d r \\
& \geq C \sigma^{-\epsilon} h_{1}(\sigma) \int_{0}^{\sigma} r^{-\frac{N+2}{q}+N-1+\epsilon} d r \geq C \sigma^{N-\frac{N+2}{q}} h_{1}(\sigma)
\end{aligned}
$$

for all sufficiently small $\sigma>0$. This implies that

$$
\int_{0}^{t} \tau^{-1}\left[\frac{\mu(B(x, \tau))}{\tau^{N-\frac{N+2}{q}}}\right]^{q} d \tau \geq \int_{0}^{t} \tau^{-1} h_{1}(\tau)^{q} d \tau
$$

for all sufficiently small $t>0$. On the other hand, since $h_{1}$ is positive continuous function in $(0,1)$, if

$$
\int_{0}^{1} \tau^{-1} h_{1}(\tau)^{q} d \tau=\infty
$$

then

$$
\int_{0}^{t} \tau^{-1} h_{1}(\tau)^{q} d \tau=\infty \text { for } t \in(0,1)
$$

Furthermore, for any $r>0$, by Lemma 2.4 we have

$$
\sup _{x \in \mathbf{R}^{N}} \nu(B(x, 1)) \leq C \sup _{x \in \mathbf{R}^{N}} \nu(B(x, r)) .
$$

Then, under the assumptions of assertion (d), we see a contradiction between (8.4), (8.5), (8.6) and assertion (3) of Theorem 1.3. Thus assertion (d) follows.

Case (E): Since $q=1+2 / N$, by (1.16) we have

$$
\int_{B(0, \sigma)} \mu(x) d x \geq C \int_{0}^{\sigma} \tau^{-1} h_{2}(\tau) d \tau
$$

for all sufficiently small $\sigma>0$. This implies that

$$
\int_{0}^{t} \tau^{-1} \mu(B(x, \tau))^{q} d \tau \geq C \int_{0}^{t} r^{-1}\left[\int_{0}^{r} \tau^{-1} h_{2}(\tau) d \tau\right]^{q} d r
$$


for all sufficiently small $t>0$. On the other hand, since $h_{2}$ is positive continuous function in $(0,1)$, if

$$
\int_{0}^{1}\left[\int_{0}^{r} \tau^{-1} h_{2}(\tau) d \tau\right]^{q} r^{-1} d r=\infty
$$

then

$$
\int_{0}^{t}\left[\int_{0}^{r} \tau^{-1} h_{2}(\tau) d \tau\right]^{q} r^{-1} d r=\infty \quad \text { for } t \in(0,1) .
$$

Furthermore, (8.6) holds. Therefore, under the assumptions of assertion (e), we see a contradiction between (8.6), (8.7), (8.8) and assertion (4) of Theorem 1.3. Thus assertion (e) follows. Furthermore, assertion (f) also follows from Lemma 2.4 and Theorem 1.1 (see also Remark 1.2). Therefore the proof of Corollary 1.2 is complete.

Acknowledgements. The first author was supported partially by the Grant-in-Aid for Early-Career Scientists (No. 19K14569). The second author of this paper was supported in part by the Grant-in-Aid for Scientific Research (S)(No. 19H05599) from Japan Society for the Promotion of Science.

\section{References}

[1] G. Akagi, K. Ishige and R. Sato, The Cauchy problem for the Finsler heat equation, Adv. Calc. Var. 13 (2020), 257-278.

[2] D. Andreucci and E. DiBenedetto, On the Cauchy problem and initial traces for a class of evolution equations with strongly nonlinear sources, Ann. Scuola Norm. Sup. Pisa Cl. Sci. 18 (1991), 363-441.

[3] D. Andreucci, M. A. Herrero and J. J. L. Velázquez, Liouville theorems and blow up behaviour in semilinear reaction diffusion systems, Ann. Inst. H. Poincaré Anal. Non Linéaire, 14 (1997), 1-53.

[4] D. G. Aronson, Non-negative solutions of linear parabolic equations, Ann. Scuola Norm. Sup. Pisa 22 (1968), 607-694.

[5] D. G. Aronson and L. A. Caffarelli, The initial trace of a solution of the porous medium equation, Trans. Amer. Math. Soc. 280 (1983), 351-366.

[6] P. Baras and M. Pierre, Critère d'existence de solutions positives pour des équations semi-linéaires non monotones, Ann. Inst. H. Poincaré Anal. Non Linéaire 2 (1985), $185-212$.

[7] P. Bénilan, M. G. Crandall and M. Pierre, Solutions of the porous medium equation in $\mathbf{R}^{N}$ under optimal conditions on initial values, Indiana Univ. Math. J. 33 (1984), 51-87.

[8] M. F. Bidaut-Véron, E. Chasseigne and L. Véron, Initial trace of solutions of some quasilinear parabolic equations with absorption, J. Funct. Anal. 193 (2002), 140-205. 
[9] M. Bonforte, Y. Sire and J. L. Vázquez, Optimal existence and uniqueness theory for the fractional heat equation, Nonlinear Anal. 153 (2017), 142-168.

[10] E. DiBenedetto and M. A. Herrero, On the Cauchy problem and initial traces for a degenerate parabolic equation, Trans. Amer. Math. Soc. 314 (1989), 187-224.

[11] E. DiBenedetto and M. A. Herrero, Nonnegative solutions of the evolution $p$-Laplacian equation. Initial traces and Cauchy problem when $1<p<2$, Arch. Rational Mech. Anal. 111 (1990), 225-290.

[12] M. Escobedo and M. A. Herrero, A uniqueness result for a semilinear reactiondiffusion system, Proc. Amer. Math. Soc. 112 (1991), 175-185.

[13] M. Escobedo and M. A. Herrero, Boundedness and blow up for a semilinear reactiondiffusion system, J. Differential Equations 89 (1991), 176-202.

[14] L. C. Evans and R. F. Gariepy, Measure Theory and Fine Properties of Functions, Studies in Advanced Mathematics, CRC Press, Boca Raton, FL, 1992.

[15] M. Fila, H. A. Levine and T. Uda, A Fujita-type global existence-global non-existence theorem for a system of reaction diffusion equations with differing diffusivities, Math. Methods Appl. Sci. 17 (1994), 807-835.

[16] Y. Fujishima and K. Ishige, Blowing up solutions for nonlinear parabolic systems with unequal elliptic operators, J. Dynam. Differential Equations 32 (2020), 1219-1231.

[17] Y. Fujishima and K. Ishige, Optimal singularities of initial functions for solvability of a semilinear parabolic system, to appear in J. Math. Soc. Japan.

[18] M. A. Herrero and M. Pierre, The Cauchy problem for $u_{t}=\Delta u^{m}$ when $0<m<1$, Trans. Amer. Math. Soc. 291 (1985), 145-158.

[19] K. Hisa and K. Ishige, Existence of solutions for a fractional semilinear parabolic equation with singular initial data, Nonlinear Anal. 175 (2018), 108-132.

[20] K. Hisa and K. Ishige, Solvability of the heat equation with a nonlinear boundary condition, SIAM J. Math. Anal. 51 (2019), 565-594.

[21] K. Ishige, On the existence of solutions of the Cauchy problem for a doubly nonlinear parabolic equation, SIAM J. Math. Anal. 27 (1996), 1235-1260.

[22] K. Ishige, T. Kawakami and M. Sierżȩga, Supersolutions for a class of nonlinear parabolic systems, J. Differential Equations 260 (2016), 6084-6107.

[23] K. Ishige and J. Kinnunen, Initial trace for a doubly nonlinear parabolic equation, J. Evol. Equ. 11 (2011), 943-957.

[24] T.-Y. Lee and W.-M. Ni, Global existence, large time behavior and life span of solutions of a semilinear parabolic Cauchy problem, Trans. Amer. Math. Soc. 333 (1992), $365-378$. 
[25] N. Mahmoudi, P. Souplet and S. Tayachi, Improved conditions for single-point blowup in reaction-diffusion systems, J. Differential Equations 259 (2015), 1898-1932.

[26] M. Marcus and L. Véron, Initial trace of positive solutions of some nonlinear parabolic equations, Comm. Partial Differential Equations 24 (1999), 1445-1499.

[27] P. Quittner and P. Souplet, Admissible $L_{p}$ norms for local existence and for continuation in semilinear parabolic systems are not the same, Proc. Roy. Soc. Edinburgh 131 (2001), 1435-1456.

[28] P. Quittner and P. Souplet, Superlinear Parabolic Problems, Blow-up, Global Existence and Steady States, Birkhäuser Advanced Texts: Basler Lehrbücher, Birkhäuser Verlag, Basel, 2007.

[29] P. Souplet, Single-point blow-up for a semilinear parabolic system, J. Eur. Math. Soc. 11 (2009), 169-188.

[30] D. V. Widder, Positive temperatures on an infinite rod, Trans. Amer. Math. Soc. 55 (1944), 85-95.

[31] H. Zaag, A Liouville theorem and blowup behavior for a vector-valued nonlinear heat equation with no gradient structure, Comm. Pure Appl. Math. 54 (2001), 107-133.

[32] J. Zhao, On the Cauchy problem and initial traces for the evolution $p$-Laplacian equations with strongly nonlinear sources, J. Differential Equations 121 (1995), 329383.

[33] J. Zhao and Z. Xu, Cauchy problem and initial traces for a doubly nonlinear degenerate parabolic equation, Sci. China 39 (1996), 673-684. 